\title{
ENREGISTREMENTS MALACOLOGIQUES À HAUTE RÉSOLUTION DANS LES LESS ET LES GLEYS DE TOUNDRA DU PLÉNIGLACIAIRE WEICHSELIEN SUPÉRIEUR: PREMIERS EXEMPLES DU NORD DE LA FRANCE
}

\author{
Olivier MOINE ${ }^{1}$, Pierre ANTOINE ${ }^{1}$, Laurent DESCHODT ${ }^{1,2}$ \\ \& Nathalie SELLIER-SEGARD ${ }^{3}$
}

\begin{abstract}
RÉSUMÉ
Les dépôts lœssiques du Pléniglaciaire supérieur weichselien ( 35-17 ka BP) d'Europe de l'ouest et centrale sont constitués par des dépôts de lœss calcaires entrecoupés par plusieurs niveaux de gleys de toundra. Leur extension continentale implique une formation liée à des variations millénaires du climat global, i.e. cycles de Dansgaard-Oeschger. L'analyse à haute résolution de la composition des malacofaunes terrestres de Nussloch a permis la caractérisation paléoenvironnementale de ces alternances sédimentaires en Allemagne dans la moyenne vallée du Rhin. Dans le présent travail, deux enregistrements malacologiques à haute résolution ont été réalisés dans le nord de la France qui appartient à un domaine malaco-biogéographique moins diversifié que celui de la vallée du Rhin. Les analyses ont permis la caractérisation de plusieurs phases environnementales associées aux alternances lœss-gleys, et la mise en évidence d'une dynamique environnementale propre à la formation de chaque gley de toundra. Des corrélations sont proposées avec les phases stadiaires et interstadiaires des cycles de Dansgaard-Oeschger, avec la stratigraphie synthétique des dépôts lœssiques du nord de la France et avec les gleys de toundra de Nussloch. Le manque de datations radiométriques associées à ces nouvelles données malacologiques reste toutefois le principal frein à la proposition d'un cadre chrono-environnemental détaillé pour ces enregistrements dans le nord de la France.
\end{abstract}

Mots-clés : malacofaunes, paléoenvironnement, lœss, gleys de toundra, Pléniglaciaire supérieur weichselien, France

\section{ABSTRACT}

HIGH RESOLUTION MOLLUSCAN RECORDS IN UPPER WEICHSELIAN LOESS AND TUNDRA GLEYS: FIRST EXAMPLES FROM NORTHERN FRANCE

West- and Central European Weichselian Upper Pleniglacial loessic deposits (35-17 ka BP) include alternations of loess layers and tundra gleys. Their continental extension implies a formation linked to millennial-timescale global climatic changes, i.e. Dansgaard-Oeschger cycles. The high resolution analysis of the composition of terrestrial malacofaunas from Nussloch allowed the palaeoenvironmental characterization of these sedimentary alternations in the Middle Rhine Valley in Germany. In the present work, two high resolution molluscan records have been performed in northern France, which belongs to a less diversified malaco-biogeographical domain than the Rhine Valley. Results show several environmental phases associated with loess-gley alternations and specific environmental dynamics during the formation of each tundra gley. Correlations are proposed with stadial and interstadial phases of Dansgaard-Oeschger cycles, with the synthetic stratigraphy of northern France loess and with Nussloch tundra gleys. The lack of radiometric ages accompanying these molluscan data is still the main limitation to proposing a detailed chrono-environmental frame for these records in northern France.

Key-words: malacofauna, palaeoenvironment, loess, tundra gleys, Weichselian Upper Pleniglacial, France

\section{1 - INTRODUCTION}

Les plaines du nord de la France se distinguent par la présence d'une couverture subcontinue de dépôts lœssiques de la dernière glaciation (Weichselien). Au sein de ces dépôts, le Pléniglaciaire supérieur est représenté par un ensemble de lœss calcaires atteignant localement jusqu'à $6 \mathrm{~m}$ d'épaisseur, et incluant plusieurs horizons repères périglaciaires présents au niveau régional. Sur la base de corrélations stratigraphiques de proche en proche d'environ une centaine de profils, étudiés notamment à l'occasion de sondages et de fouilles archéologiques, une séquence synthétique comportant un maximum de trois unités principales séparées par des niveaux repères de type gley de toundra cryoturbé ou géliflué a été définie puis progressivement complétée (Antoine, 1990; Antoine et al., 1998, 2003) (fig. 1).

\footnotetext{
${ }^{1}$ Laboratoire de Géographique Physique, UMR 8591 CNRS - Universités Paris 1-4-7-12, 1 place Aristide Briand, F-92195 MEUDON cedex. Courriel: olivier.moine@cnrs-bellevue.fr

${ }^{2}$ INRAP Centre Nord-Picardie - ZI La Pilaterie, 11 rue des Champs, F-59650 VILLENEUVE D’ASCQ. Courriel: laurent.deschodt@inrap.fr

${ }^{3}$ INRAP Centre Archéologique de Guadeloupe, route de Dolé, 97113 GOURBEYRE. Courriel: nathalie.sellier-segard@inrap.fr
} 


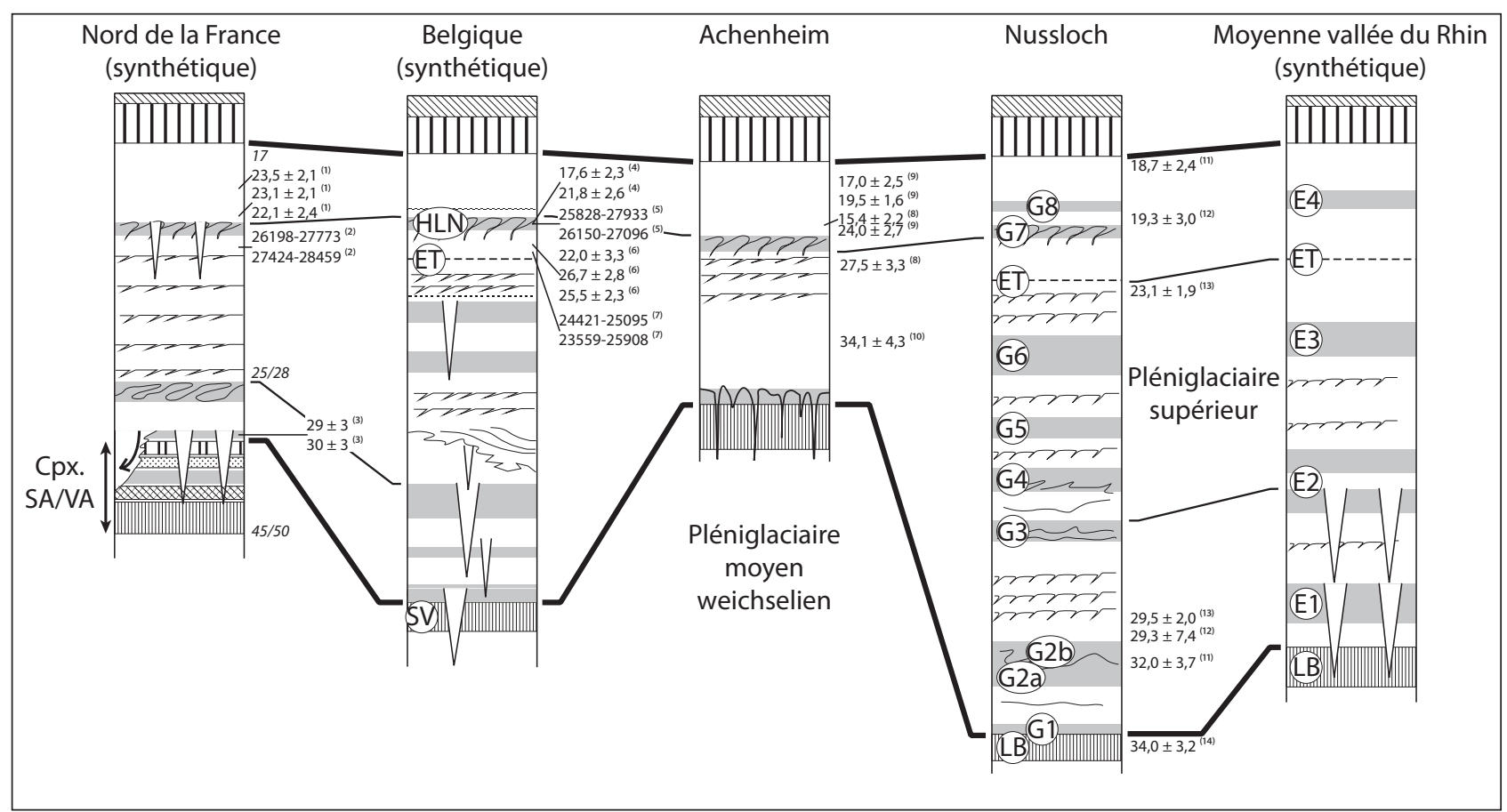

Fig. 1: Chronologie numérique et corrélation des séquences synthétiques du Pléniglaciaire supérieur weichselien du nord de la France, de Belgique, de la vallée du Rhin, d'Achenheim et de Nussloch, d'après Antoine et al. (2001) modifié.

Principales unités stratigraphiques: complexe de sols de Saint-Acheul / Villiers-Adam (Cpx. SA/VA), sol des Vaux (SV), Lohner Boden (LB), tuf d'Eltville (ET), gleys de toundra (G1 à G8), Erbenheimer Boden (E1 à E4) et horizon à langues de Nagelbeek (HLN). Datations: 1) Locht et al. (2003) - TL, TL et IRSL (de haut en bas); 2) Fagnart (inédit) $-{ }^{14} \mathrm{C} 22360 \pm 240$ cal. BP (sup.) et $23040 \pm 220$ BP (inf.) calibrées avec Calib 6.0 (Reimer et al., 2009); 3) Locht et al. (2006) - ESR os (sup.), ESR dent (inf.); 4) van den Haute et al. (1998) - TL; 5) Gullentops (1981) - ${ }^{14}$ C $22270 \pm 380$ cal. BP (sup.) et $22190 \pm 130$ cal. BP (inf.) calibrées avec Calib 6.0 (Reimer et al., 2009); 6) Frechen et al. (2001) - IRSL regeneration (sup.), TL regeneration (milieu) et TL additive (inf.); 7) Vandenberghe et al. (1998) $-{ }^{14} \mathrm{C} 20780 \pm 120$ cal. BP (sup.) et $20660 \pm 460$ cal. BP (inf.) calibrées avec Calib 6.0 (Reimer et al., 2009); 8) Buraczynski et Butrym (1984) - TL; 9) Rousseau et al. (1998) - TL additive; 10) Zöller et al. (2004) - TL; 11) Bibus et al. (2007) - IRSL; 12) Rousseau et al. (2007) - IRSL; 13) Tissoux et al. (2010) - OSL; 14) Lang et al. (2003) - IRSL.

Fig. 1: Radiochronology and correlation between Weichselian Upper Pleniglacial synthetic sequences from northern France, Belgium, Rhine Valley, Achenheim and Nussloch, after Antoine et al. (2001) modified. Main stratigraphical units: Saint-Acheul / Villiers-Adam soil complex (Cpx. SA/VA), Les Vaux soil (SV), Lohner Boden (LB), Eltville tuff (ET), tundra gleys (G1 to G8), Erbenheimer Boden (E1 to E4) et Nagelbeek tongue horizon (HLN). Datings: 1) Locht et al. (2003) - TL, TL and IRSL (from top to bottom); 2) Fagnart (unpublished) - ${ }^{14} \mathrm{C} 22360 \pm 240$ cal. BP (sup.) and $23040 \pm 220$ cal. BP (inf.) calibrated with Calib 6.0 (Reimer et al., 2009); 3) Locht et al. (2006) - ESR bone (sup.), ESR tooth (inf.); 4 ) van den Haute et al. (1998) - TL; 5) Gullentops (1981) $-{ }^{14} \mathrm{C} 22270 \pm 380$ cal. BP (sup.) and $22190 \pm 130$ cal. BP (inf.) calibrated with Calib 6.0 (Reimer et al., 2009); 6) Frechen et al. (2001) - IRSL regeneration (sup.), TL regeneration (middle) et TL additive (inf.); 7) Vandenberghe et al. (1998) $-{ }^{14} \mathrm{C} 20780 \pm 120$ cal. $B P$ (sup.) and $20660 \pm 460 \mathrm{cal}$. BP (inf.) calibrated with Calib 6.0 (Reimer et al., 2009); 8) Buraczynski and Butrym (1984) - TL; 9) Rousseau et al. (1998) - TL additive; 10) Zöller et al. (2004) - TL; 11) Bibus et al. (2007) - IRSL; 12) Rousseau et al. (2007) - IRSL; 13) Tissoux et al. (2010) - OSL; 14) Lang et al. (2003) - IRSL.

$\mathrm{Au}$ Pléniglaciaire supérieur deux domaines malacobiogéographiques, nommés «ouest» et «central», ont été différenciés en Europe de l'ouest sur la base des malacofaunes des dépôts lœssiques (Moine, 2008). D'après la valence écologique de ses taxons, le domaine «ouest», qui s'étend sur les régions périphériques à la Manche dont la moitié ouest du nord de la France, est plus humide et à végétation pauvre. Le domaine «central», qui s'étend du Bassin Parisien à l'est de l'Allemagne, est quant à lui plus sec et à végétation plus diversifiée (fig. 2).

D'après les datations numériques disponibles, les dépôts lœssiques du Pléniglaciaire supérieur se sont mis en place entre environ 35 ka BP (âge moyen du dernier sol brun arctique/boréal du Pléniglaciaire moyen weichselien) et environ 17 ka BP (âge moyen d'arrêt de la sédimentation lœssique en Europe) (fig. 1) (Antoine et al., 2001 ; Vandenberghe et al., 2004). Entre ces deux âges limites, la plupart des enregistrements malacologiques sont discontinus, et restent mal datés (absence de datations numériques). La reconstitution précise de l'évolution paléoenvironnementale spatiale et temporelle n'est donc actuellement pas possible pour cette période.
Ainsi, dans le domaine «central», seuls les enregistrements malacologiques de Hainerberg et Gräselberg (Wiesbaden, Allemagne) (Remy, 1969), Schwalbenberg (Remagen, Allemagne) (Schiermeyer, 2002) et Nussloch (Allemagne) (Moine et al., 2008) ont fait l'objet d'un échantillonnage selon une colonne continue avec une résolution suffisante pour aborder la dynamique environnementale à l'échelle millénaire. Dans le domaine «ouest», la plupart des enregistrements malacologiques incluent moins de cinq échantillons prélevés isolément, et au mieux dans les différentes unités stratigraphiques (Moine, 2008). A Halling (Royaume-Uni) (Kerney, 1971) et à Maisières-Canal (Belgique) (de Coninck, 1973), 25 et 12 échantillons ont bien été prélevés respectivement en colonne continue, et en colonne discontinue. Toutefois, les alternances sédimentaires de ces deux sites ne sont pas typiques des dépôts lœssiques régionaux en raison de conditions de dépôt particulières (fond de vallée à Maisières-Canal et dépôts de versants crayeux à matrice lœssique à Halling).

La dynamique malacologique et environnementale millénaire du Pléniglaciaire supérieur reste donc mal 


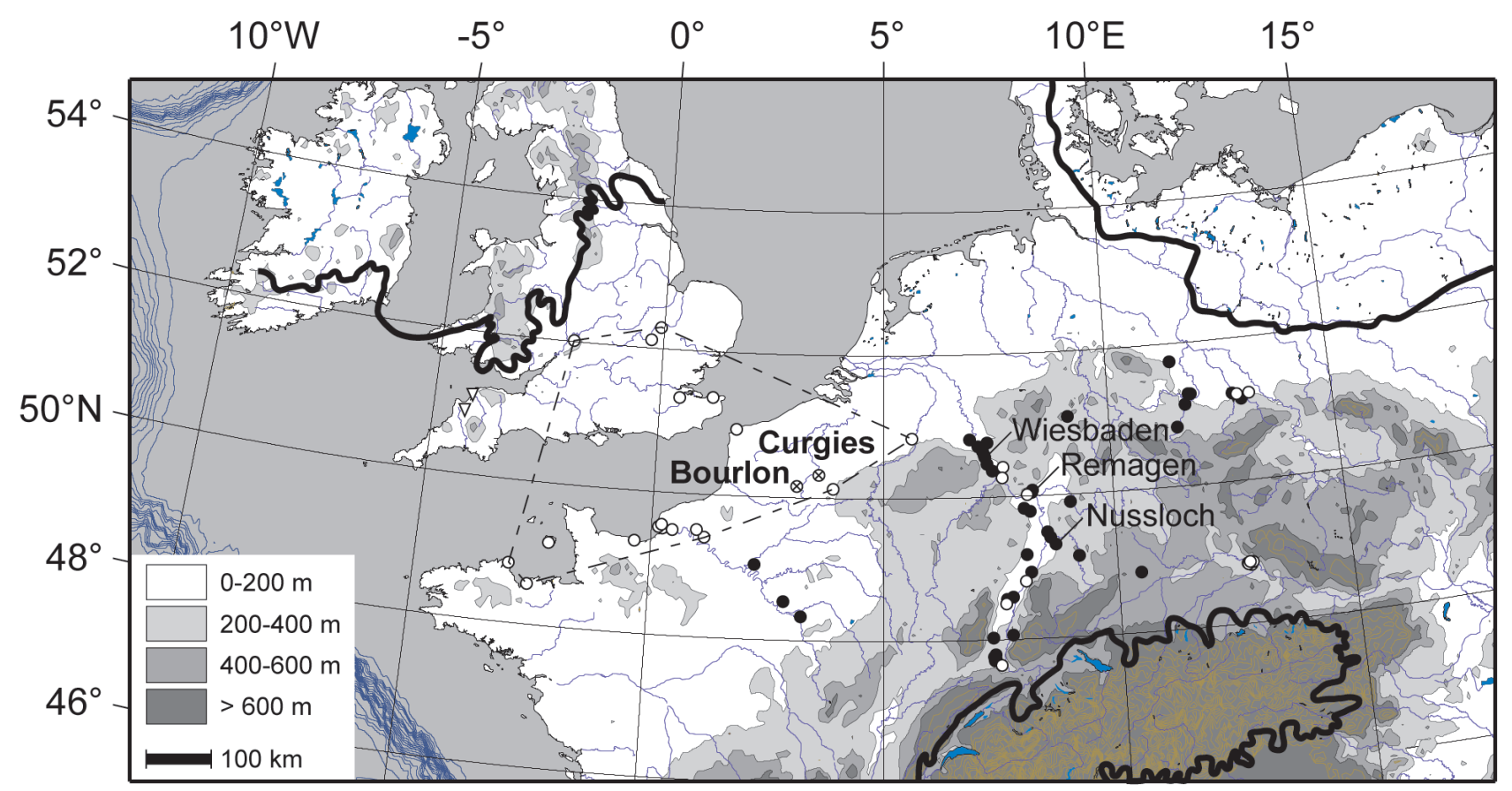

Fig. 2 : Localisation des nouveaux sites échantillonnés (cercles à croix) dans le domaine malaco-biogéographique « ouest» (ligne pointillée) et des enregistrements malacologiques à haute résolution disponibles dans le domaine «central». Les cercles blancs indiquent les sites à malacofaune pauvre (1-6 taxons parmi Columella columella, Pupilla muscorum, Succinella oblonga, Trochulus hispidus, Oxyloma elegans, limaces). Les cercles noirs indiquent les sites à malacofaune plus diversifiée.

Fig. 2: Location of newly sampled sites (circles with a cross) in the "western» malaco-biogeographical domain (dashed line) and available high resolution molluscan records in the «central» domain. White circles indicate sites with a poor malacofaune (1-6 taxa among Columella columella, Pupilla muscorum, Succinella oblonga, Trochulus hispidus, Oxyloma elegans, slugs). Black circles indicate sites with a richer malacofauna.

connue, notamment dans le domaine «ouest». Les nouveaux enregistrements à haute résolution réalisés dans le cadre de sondages liés à des opérations d'archéologie préventive ont donc fait l'objet de l'étude qui va suivre.

\section{2 - CONTEXTE SÉDIMENTAIRE ET PALÉOENVIRONNEMENTAL}

Les dépôts lœssiques du Pléniglaciaire supérieur présentent les plus forts taux de sédimentation de la dernière glaciation (Frechen et al., 2003) et sont formés d'alternances de niveaux de lœss et de gleys de toundra visibles dans la plupart des profils du nord-ouest de l'Europe continentale (Semmel, 1968; Bibus, 1980; Haesaerts, 1980; Antoine, 1988; Huijzer, 1993 ; Frechen, 1999; Frechen et al., 1999; Schirmer, 2000; Antoine, 2002; Vandenberghe et al., 2004; Antoine et al., 2009). Dans la majorité des sites étudiés, deux horizons repères sont presque toujours présents. A la base des séquences, le «sol de Saint-Acheul / Villiers-Adam», aussi appelé «sol des Vaux» en Belgique et «Lohner Boden» en Allemagne, représente le dernier sol brun arctique/boréal du Pléniglaciaire moyen weichselien (fig. 1). Le terme «complexe de sols de Saint-Acheul / Villiers-Adam» est utilisé dans les profils où ce sol ne peut pas être distingué des autres horizons du Pléniglaciaire moyen. Vers le sommet des séquences, «l'horizon à langues de Nagelbeek», aussi appelé «Erbenheimer Boden E4» en Allemagne, est le dernier gley de toundra précédant les dépôts de lœss homogène souvent épais du Dernier Maximum Glaciaire (lœss Brabantien; Haesaerts et al., 1981). Entre ces deux niveaux repères, plusieurs horizons du type gley de toundra alternent avec des niveaux de lœss lité à microfentes de cryodessication liés à une dynamique nivéo-éolienne (Antoine et al., 2002) et (ou) localement, dans les coupes situées à proximité d'une importante source de sables, à un litage purement éolien comme à Nussloch en rive droite de la vallée du Rhin (Antoine et al., 2009).

La dénomination «gley de toundra» désigne des horizons hydromorphes résultant de la saturation en eau de la couche active d'un pergélisol riche en glace au cours de périodes de dégel estival. Ils sont caractérisés par une teinte générale grisâtre à verdâtre due à la réduction du fer dans le sédiment, ainsi que par la présence de bandes irrégulières et de tâches de couleur rouille résultant de processus d'oxydation localisés au niveau des traces de racines et de bioturbations diverses (tubules à auréoles oxydées de quelques millimètres de diamètre). On note également la présence d'un léger enrichissement en carbone organique dû à un enracinement plus dense, une activité biologique plus intense et un léger enrichissement en argile (+ 4 à $6 \%$ ) par rapport aux lœss encaissants (Antoine et al., 2009). Enfin, une faible décalcification peut redistribuer une partie des carbonates à la base de l'horizon sous la forme de petites concrétions calcaires (poupées du lœss) (Huijzer, 1993; Antoine et al., 2001).

Au cours des multiples alternances gel-dégel qui affectent ces horizons, des processus de cryoturbation (cryoinjection) affectent les gleys de toundra formés en contexte 
de plateau ou de faible pente et produisent des petits polygones de toundra (maille $20-30 \mathrm{~cm}$ ) bien visibles en décapage horizontal. Lors des phases de dégradation particulièrement marquées du pergélisol, les involutions subissent des processus de fluage lent (cryoreptation; Van Vliet-Lanoë, 1985) qui produisent une déformation des structures dans le sens de la pente et la formation des «horizons à langues». Dans les sites situés sur pente, ce processus débouche sur un fluage généralisé de plus grande ampleur de la couche active (gélifluxion) et génère une érosion des niveaux sous-jacents.

Dans les séquences du nord de la France, de grandes fentes en coin de type "ice-wedge», de 1 à 2 mètres de profondeur pour 0,5 à 0,6 mètres d'ouverture, sont systématiquement associées aux horizons de gley de toundra (Antoine, 1990; 1991). Le fluage de la partie supérieure de la couche active du gley de toundra à l'intérieur de la fente en cours de dégradation (fonte de la glace du coin) démontre une relation intime entre le fonctionnement des réseaux de grande fente et des gleys de toundra, comme c'est actuellement le cas dans les environnements à sols polygonaux (French, 2007), ou comme Haesaerts et Van Vliet-Lanoë (1974) l'ont observé dans les dépôts lœssiques de Belgique.

Les alternances lœss-gley peuvent être très semblables entre les différents profils d'une même région, comme par exemple dans la vallée du Rhin (Semmel, 1968; Bibus, 1980) ou dans le nord de la France (Antoine, 1991). Les niveaux de lœss et les gleys de toundra ont alors les mêmes positions stratigraphiques et parfois des épaisseurs et des signatures sédimentaires très similaires. Une telle homogénéité des signatures stratigraphiques implique que la formation des gleys de toundra dans les dépôts lœssiques résulte de changements climatiques d'envergure continentale et en aucun cas de facteurs locaux.

Dans le nord de la France comme dans la vallée du Rhin, la présence systématique de faciès lités nivéoéoliens à microfentes dans la partie inférieure du Pléniglaciaire supérieur confirme la mise en évidence d'une tonalité régionalement plus humide (rôle de la couverture nivale) qu'au moment de la mise en place des lœss calcaires homogènes postérieurs à l'horizon à langues de Nagelbeek qui traduisent un environnement particulièrement sec et aride. La partie inférieure des dépôts de lœss lité nivéo-éolien du Pléniglaciaire supérieur inclut généralement plusieurs gleys de toundra épais $(\sim 0,5 \mathrm{~m})$ et cryoturbés. Ils sont beaucoup plus ténus et faiblement déformés dans la partie supérieure, excepté l'horizon à langues de Nagelbeek, qui présente un faciès typique à langues grisâtres humifères dans une zone côtière allant de la Normandie à la Belgique, et qui peut-être considéré comme le plus récent des gleys de toundra pléniglaciaires (Haesaerts, 1985 ; Van Vliet-Lanoë, 1992 ; Huijzer, 1993 ; Vandenberghe et al., 1998).

Parallèlement à cette règle générale, il existe une variabilité importante concernant la puissance des dépôts lœssiques du Pléniglaciaire supérieur, ainsi que le nombre et l'aspect des gleys de toundra. Par exemple, on a d'une part le cas unique de Nussloch, où un maximum de huit gleys de toundra (dont cinq principaux et deux biphasés) ont été observée dans une séquence de plus de treize mètres d'épaisseur (Antoine et al., 2009), et d'autre part de nombreux profils ne montrant pas plus de trois ou quatre gleys de toundra dans une séquence dépassant rarement trois ou quatre mètres d'épaisseur (Antoine, 1990, 1991). Malgré un forçage climatique d'envergure continentale, à l'origine de la formation des gleys de toundra et du dépôt des différentes unités de lœss, des différences significatives ont été notées entre les séquences. Ces dernières peuvent résulter de variations importantes du taux de sédimentation en liaison avec les conditions géomorphologiques locales et la distances aux sources, et (ou) de phases d'érosion conduisant à la présence de hiatus.

Lorsque deux phases de formation de gley de toundra ne sont séparées que par un mince dépôt de lœss, ce dernier est affecté sur toute son épaisseur par la formation du gley de toundra supérieur. Les deux gleys de toundra apparaissent alors sous la forme d'une seule unité complexe incluant deux phases de cryoturbation et de gleyification en partie superposées et visuellement indissociables. C'est le cas du gley de toundra G2 à Nussloch dans les profils P2 et P3 (Antoine et al., 2002, 2009) et très vraisemblablement dans les séquences du nord de la France au niveau de l'Horizon cryoturbé du Santerre (Antoine, 1991 ; Antoine, obs. pers.).

Par ailleurs, des phases d'érosion ont pu conduire à la disparition de tout ou partie des sédiments préalablement déposés. Des datations précises sont alors requises pour évaluer les hiatus potentiels. Dans les coupes du bassin de la Somme comme Saint-Sauflieu ou Renancourt par exemple, la sédimentation au Pléniglaciaire supérieur débute directement par des lœss lités sans aucune trace de gley de toundra (Antoine, 1990; Fagnart et al., sous presse). Toujours à Saint-Sauflieu, l'horizon à langues de Nagelbeek est remplacé par un niveau d'érosion souligné par un cailloutis cryoturbé dont la mise en place est liée à un contexte plus pentu et à l'affleurement d'un substrat caillouteux sur le versant surplombant le site.

L'interprétation paléoenvironnementale des alternances lœss-gley du Pléniglaciaire supérieur a récemment progressé grâce à des analyses granulométriques (Antoine et al., 2001; Rousseau et al., 2002, 2007; Antoine et al., 2009), géochimiques (Hatté et al., 1999, 2001) et malacologiques (Moine et al., 2008) à haute résolution réalisées dans les dépôts lœssiques de Nussloch. Ces travaux ont permis de proposer des corrélations entre les alternances lœss-gley les plus marquées et les variations climatiques cycliques millénaires de la dernière glaciation, i.e. cycles de Dansgaard-Oeschger, qui ont débouché sur le modèle conceptuel suivant (fig. 3):

A-Stade: taux de sédimentation lœssique élevé, taille élevée des particules, malacofaunes peu abondantes Environnement froid et sec.

B- Transition stade-interstade: baisse du taux de sédimentation lœssique, taille décroissante des particules, mise en place d'un pergélisol, développement d'un gley de toundra, ouverture de coins de glace sous la couche active, et malacofaunes peu abondantes, accroissement des proportions en taxons hygrophiles et palustres Environnement froid et plus humide. 


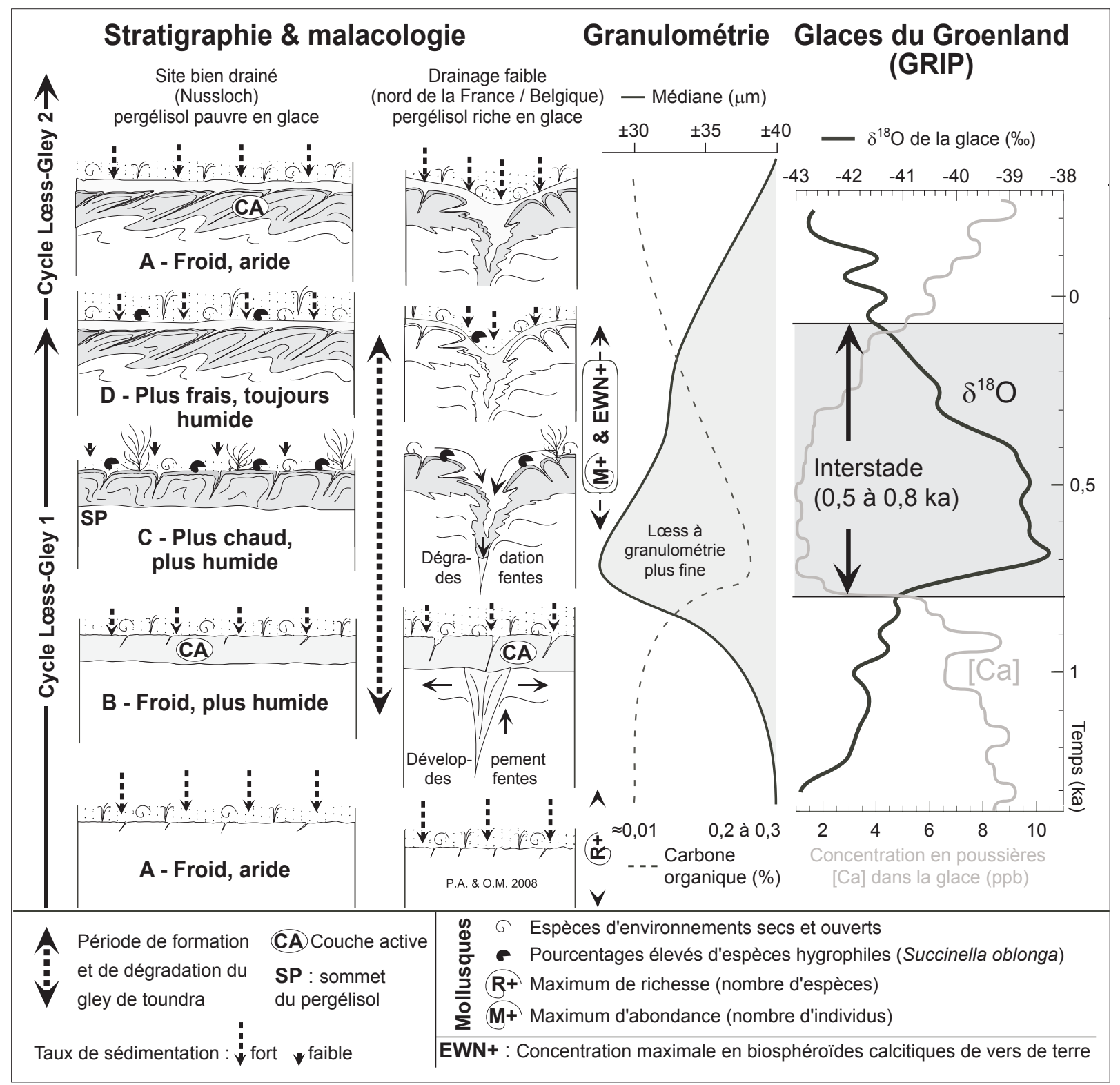

Fig. 3 : Modèle conceptuel reliant les alternances loss-gley et l'évolution pédologique, aux changements de composition de la malacofaune, et aux variations millénaires de type stade-interstade des indicateurs du climat global des glaces du Groenland, d'après Antoine et al. (2009) modifié.

Fig. 3: Conceptual model linking loess-gley alternations and their pedological evolution to composition changes of the malacofauna, and to stadialinterstadial millennial timescale variations of global climate proxies from Greenland ice-cores, from Antoine et al., (2009) modified.

C- Interstade: taille minimale des particules, approfondissement de la couche active et du gley de toundra, et proportion maximale en taxons hygrophiles et palustres, puis fonte des coins de glace, gélifluxion et (ou) cryoturbation du gley de toundra, malacofaunes très abondantes, et proportion de juvéniles morts très élevée - Environnement humide avec une saison chaude plus douce et plus longue, et des hivers toujours rigoureux.

D- Transition interstade-stade: dernières phases de gélifluxion et (ou) cryoturbation du gley de toundra, retour rapide à un fort taux de sédimentation lœssique, légère augmentation de la taille des particules, réduction drastique et brutale des populations de mollusques, diminution des proportions de taxons hygrophiles et palustres - Environnement froid et sec l'hiver, et rapidement de moins en moins humide à la saison chaude qui baisse en intensité et raccourcit en durée.
Le modèle proposé par Vandenberghe et Nugteren (2001) est proche de celui-ci et implique également l'installation d'un pergélisol en association avec l'ouverture des coins de glace et le développement des gleys de toundra. La principale différence proposée sur la base des nouvelles recherches menées à Nussloch et dans le nord de la France est un développement des gleys de toundra synchrone de la formation des grandes fentes à coins de glace et non postérieur. Les séquences observées montrent en effet un fluage systématique par gélifluxion des gleys de toundra (couche active) sur la bordure et dans les dépressions liées à la fonte des coins de glace associés. Une fois ce processus terminé, ces dépressions sont colmatées lors de la mise en place de lœss typique (fossilisation et formation des pseudomorphoses de coins de glace) dans un environnement à nouveau très froid et aride. Les analyses malacologiques 
à haute résolution associées aux séquences étudiées ont en outre permis de préciser la dynamique des variations d'humidité et de température associées à ces alternances lœss-gley.

Dans le nord de la France, la séquence stratigraphique synthétique des dépôts du Pléniglaciaire supérieur est formée depuis la base, d'un gley de toundra géliflué associé à un réseau de grands coins de glace, d'un lœss calcaire homogène d'épaisseur réduite $(\sim 0,5$ à $0,6 \mathrm{~m})$ mais extrêmement constant au niveau régional, d'un gley de toundra épais et cryoturbé (Horizon cryoturbé du Santerre), d'un lœss calcaire lité à microfentes, d'un gley de toundra géliflué grisâtre faiblement humifère (horizon à langues de Nagelbeek) souvent associé à un deuxième réseau de coins de glace dégradés (gélifluxion), et d'un lœss calcaire homogène (loess de couverture) (fig. 1).

Dans la vallée du Rhin, la base de la stratigraphie synthétique est identique avec un gley de toundra géliflué à grands coins de glace, et un lœss calcaire homogène d'épaisseur réduite. Ensuite, quatre gleys de toundra (Erbenheimer Boden E1, E2, E3 et E4), se succèdent alternant avec d'épais dépôts de lœss lité. Le plus récent de ces gleys de toundra (E4) est recouvert par un dépôt de lœss homogène comme dans le nord de la France. Le gley de toundra E2 est parfois dédoublé et des coins de glace lui sont souvent associés. Enfin, un niveau de tuf volcanique (tuf d'Eltville) est souvent présent entre les gleys de toundra E3 et E4 (fig. 1).

L'enregistrement sédimentaire du Pléniglaciaire supérieur de la séquence de Nussloch est particulièrement complet. Grâce aux nombreuses datations par luminescence (Lang et al., 2003; Rousseau et al., 2007; Antoine et al., 2009) et ${ }^{14} \mathrm{C}$ (Hatté et al., 2001) composant sa chronologie numérique, des corrélations précises ont été proposées entre les alternances lœss-gley de cette séquence et les variations des indicateurs climatiques globaux de la calotte de glace arctique, notamment ceux de la concentration en poussière. Toutefois, mis à part les séquences de l'est des Carpates où de nombreuses datations ${ }^{14} \mathrm{C}$ ont été obtenues à partir de charbons de bois provenant d'horizons essentiellement archéologiques (Haesaerts et al., 2003), bien peu de profils stratigraphiques disposent d'un tel cadre chronologique. La datation des alternances lœss-gley et leur corrélation entre profils stratigraphiques d'une même région ou de régions différentes sont donc délicates sur la seule base de leur faciès et de leur position stratigraphique, notamment en raison des variations du taux de sédimentation et de la présence de hiatus d'origine érosive.

L'objectif de cette étude est donc la caractérisation malacologique des changements paléoenvironnementaux associés aux alternances lœss-gley du Pléniglaciaire supérieur dans le nord de la France, et leur comparaison avec ceux de la vallée du Rhin. En parallèle, nous avons cherché à déterminer si les alternances lœss-gley étaient caractérisées par une dynamique des populations de mollusques terrestres propre à chacune, pouvant constituer un élément de corrélation entre profils stratigraphiques du nord-ouest de l'Europe.

\section{3 - NOUVELLES DONNÉES}

\section{1 - LOCALISATION DES SITES}

Le matériel malacologique analysé dans ce travail provient de deux séquences lœssiques situées dans le nord de la France à Curgies (Nord), environ sept kilomètres à l'est de Valenciennes et à Bourlon (Pas-de-Calais), environ huit kilomètres à l'ouest de Cambrai (fig. 2). Les séquences sédimentaires de ces deux sites ont été observées à la faveur de sondages de diagnostics archéologiques opérés par l'Institut National de Recherches Archéologiques Préventives (INRAP).

A Curgies, la zone sondée se situe en position d'interfluve sur les plateaux les plus septentrionaux du Bassin Parisien, en rive droite de l'Escaut. Le sondage SP2 a fait l'objet d'un levé stratigraphique, de prélèvements malacologiques et de trois échantillons destinés à réaliser des datations par luminescence optiquement stimulée (OSL) (fig. 4). Les résultats des datations n'étaient toutefois pas encore disponibles au moment de la rédaction de cet article.

A Bourlon, la zone sondée se situe en contexte de plateau, le cours d'eau le plus proche étant le ruisseau de l'Agache. Deux sondages (ZP7 et ZP 8) ont fait l'objet d'un levé stratigraphique et de prélèvements malacologiques (fig. 4). Les prélèvements malacologiques T1 à T4 du profil ZP7 ont été replacés sur le profil ZP8. Les prélèvements malacologiques réalisés à Bourlon n'ayant au départ qu'une valeur de test, aucune datation n'a été envisagée pour ce site.

\section{2 - CHRONOSTRATIGRAPHIE}

L'interprétation chronostratigraphique des profils de Curgies et de Bourlon s'appuie sur la synthèse des travaux réalisés dans les dépôts lœssiques du nord de la France au cours des vingt dernières années (Antoine et al., 1998, 1999, 2003). En l'absence de datations radiométriques, elle est principalement fondée sur l'analyse de la succession des unités litho-pédo-stratigraphiques des horizons périglaciaires, leur hiérarchisation et leur intégration dans le canevas stratigraphique régional (fig. 4).

Dans le profil de Curgies, le limon brun orangé de base (1) a été interprété comme un horizon Bt de sol brun lessivé interglaciaire rapporté à l'Eemien, alors que le limon brun orangé sus-jacent (2), interprété comme un sol forestier, représente quant à lui un bilan condensé de plusieurs pédogénèses du Début-Glaciaire weichselien. Le Début-Glaciaire weichselien est surtout représenté par le limon gris (3), interprété comme un sol steppique, et attribué à l'un des derniers épisodes de pédogenèse de cette longue période de transition. La plupart des artéfacts archéologiques découverts à Curgies proviennent de la partie supérieure de cet horizon humifère. Ce pédocomplexe a été observé à des profondeurs très variables dans les différents sondages réalisés, témoignant d'une paléotopographie nettement différente de l'actuelle. Le niveau repère suivant est le limon argileux brun-orangé feuilleté (9) interprété comme le sol de 


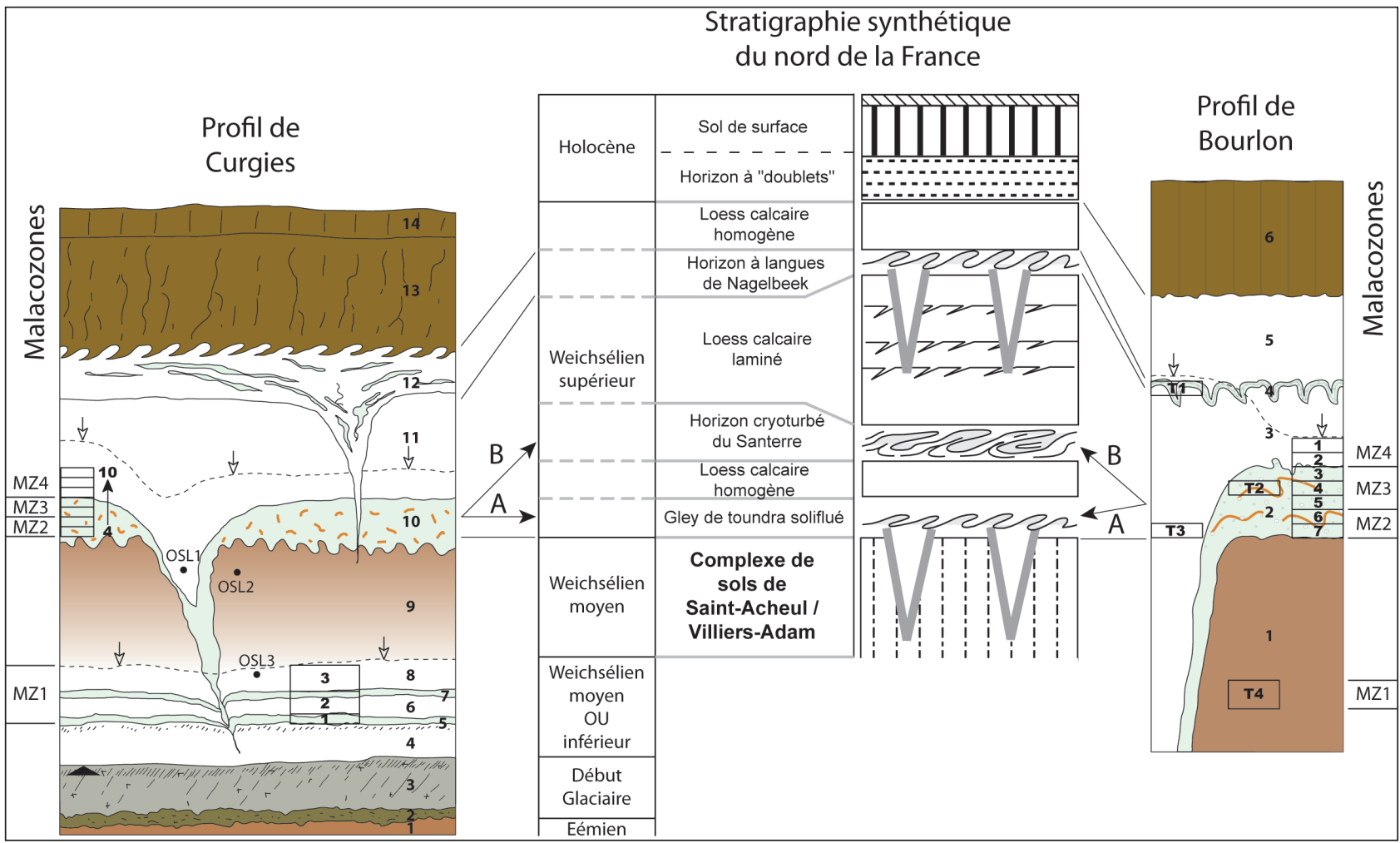

Fig. 4 : Comparaison des profils stratigraphiques de Curgies et de Bourlon avec la stratigraphie synthétique des dépôts loessiques du Pléniglaciaire supérieur weichselien du nord de la France.

Les flèches labellisées A et B indiquent les corrélations possibles du principal gley de toundra de chaque séquence. Le triangle noir indique la position des artéfacts archéologiques. Description des unités sédimentologiques de Curgies: (1) limon brun orangé structuré, (2) limon brun orangé foncé formé d'agrégats, (3) limon gris avec un horizon blanchi à la base et des tâches d'hydromorphie au sommet, (4) limon brun jaune terne non calcaire avec horizon plus sombre au sommet, (5) gley de toundra calcaire, (6) limon brun jaune calcaire à coloration rouille, (7) gley de toundra calcaire, (8) limon jaune calcaire, (9) limon brun-orangé décarbonaté à légère structuration (complexe de sols de Saint-Acheul / Villiers-Adam), (10) gley de toundra calcaire avec un coin de glace de taille métrique, (11) limon jaune calcaire décarbonaté dans sa partie supérieure, (12) limon à lambeaux de gley de toundra soliflués avec un coin de glace de taille métrique, et dont le sommet est un horizon géliflué, (13) limon brun, (14) horizon de labour. Description des unités sédimentologiques de Bourlon: (1) limon brun feuilleté avec pseudomycélium (complexe de sols de Saint-Acheul/Villiers-Adam), (2) gley de toundra calcaire avec un coin de glace de taille métrique, (3) limon jaune calcaire homogène avec grains de craie, (4) fin gley de toundra avec coins de glace de taille décimétrique, (5) limon jaune calcaire avec grains de craie, ce dépôt est partiellement ou totalement décalcifié en fonction du profil, (6) limon brun.

Fig. 4: Comparisons of stratigraphic profiles from Curgies and Bourlon with the synthetic stratigraphy of Weichselian Upper Pleniglacial loess deposits from northern France. Arrows labelled A and B show possible correlations of the main tundra gley of each sequence. The black triangle indicates the position of archaeological artifacts. Description of sedimentological units from Curgies: (1) brown orange and structured loam, (2) brown dark orange loam made of aggregates, (3) gray loan with a white horizon at the base and hydromorphic spots at the top, (4) brown yellow non calcareous loam with a darker horizon at the top, (5) calcareous tundra gley, (6) brow yellow calcareous loam with a rusty color, (7) calcareous tundra gley, (8) yellow calcareous loam, (9) brown orange decarbonated loam with slight structure (Saint-Acheul/Villiers-Adam soil complex). (10) calcareous tundra gley with a metric-size ice wedge, (11) yellow calcareous loam, the upper half decalcified, (12) soliflucted loam with shreds of tundra gley, with a metric-size ice wedge, and with a cryoturbated horizon on top, (13) brown loam, (14) plough horizon. Description of sedimentological units from Bourlon: (1) brown laminated loam with pseudomycelium (Saint-Acheul/Villiers-Adam soil complex), (2) calcareous tundra gley with a metric-size ice wedge, (3) yellow homogeneous calcareous loam with chalk grains, (4) thin tundra gley with decimetric size ice wedges, (5) yellow calcareous loam with chalk grains, this deposit is partly or totally decalcified depending on the profile, (6) brown loam.

Saint-Acheul / Villiers-Adam ou la partie supérieure du complexe de sols de Saint-Acheul / Villiers-Adam. L'ensemble de limons jaune et de gleys de toundra calcaires compris entre le complexe de sols du Début-Glaciaire et le complexe de sols de Saint-Acheul / Villiers-Adam (unités 4 à 8 ) serait donc d'âge Weichselien inférieur ou moyen. Pour confirmer son âge une datation OSL est en cours. L'ensemble de limons jaune et de gleys de toundra calcaires sus-jacents au complexe de sols de Saint-Acheul / Villiers-Adam (unités 10 à 12) est donc logiquement attribuable au Pléniglaciaire weichselien supérieur. Des datations OSL du sol de SaintAcheul / Villiers-Adam (9) et du limon jaune calcaire (11) remplissant la pseudomorphose de coin de glace sont en cours pour préciser leur âge. Bien qu'étant directement sus-jacent au complexe de sols de Saint-
Acheul / Villiers-Adam, aucun élément ne permet de préciser si le gley de toundra de base est corrélé avec le gley de toundra de base (A) ou avec l'horizon cryoturbé du Santerre (B) dans la stratigraphie synthétique des dépôts lœssiques du nord de la France (fig. 4). L'horizon géliflué qui termine cet ensemble (12) est interprété comme étant l'équivalent local de «l'horizon à langues de Nagelbeek»».

A Bourlon, seule la partie supérieure des sondages ZP7 et ZP8 a été explorée en détail. Le limon brun argileux feuilleté (1), épais de plusieurs mètres, et au sein duquel aucune subdivision n'a été mise en évidence, a été mis en parallèle avec le complexe de sols de SaintAcheul / Villiers-Adam. L'ensemble lœssique calcaire sus-jacent (unités 2 à 5) est donc logiquement d'âge Weichselien supérieur. Compte tenu du faible développe- 
ment vertical de la séquence de Bourlon, il est possible de corréler le gley de toundra (2) observé à la base des lœss calcaires soit avec le gley de toundra de base (hypothèse A), soit avec l'horizon cryoturbé du Santerre (hypothèse B) dans la stratigraphie synthétique régionale (fig. 4). En raison de sa position stratigraphique, le gley de toundra supérieur (4) est très certainement l'expression locale de l'horizon à langues de Nagelbeek.

\section{3 - PRÉLÈVEMENT ET ANALYSE DES ÉCHAN- TILLONS MALACOLOGIQUES}

A Curgies, des échantillons de 5 litres de sédiment ont été prélevés en continu dans deux colonnes distinctes (fig. 4). Au-dessous le complexe de sols de SaintAcheul / Villiers-Adam, la première colonne s'étend de la base du gley de toundra inférieur au sommet du limon jaune supérieur. De la base vers le sommet, les échantillons ont des épaisseurs de respectivement 8, 25 et $25 \mathrm{~cm}$. Au-dessus du sol de Saint-Acheul / VilliersAdam, la seconde colonne s'étend de la base du gley de toundra épais à coins de glace presque jusqu'à la limite de décarbonatation située dans le limon jaune sus-jacent. Tous les échantillons correspondent à des tranches de sédiment d'une épaisseur de $10 \mathrm{~cm}$.

A Bourlon, des échantillons de 10 litres de sédiment et de $10 \mathrm{~cm}$ d'épaisseur, excepté T4 $(20 \mathrm{~cm})$, ont été prélevés ponctuellement (T1 à T4) (fig. 4) à titre de tests dans les différentes structures pédo-sédimentaires visibles dans le sondage ZP7, à savoir le limon brun feuilleté, le gley de toundra de base, le remplissage lœssique d'une grande fente à coin de glace, et le gley de toundra supérieur à petites fentes de gel (réseau polygonal décimétrique). Les échantillons 1 à 7 ont été prélevés en colonne continue dans le sondage ZP8 depuis le sommet du complexe de sols de Saint-Acheul / Villiers-Adam jusqu'à la limite de décarbonatation qui s'enfonçait plus profondément dans ce deuxième sondage.

$\mathrm{Au}$ laboratoire, les échantillons prélevés ont été tamisés à l'eau sur un tamis de $425 \mu \mathrm{m}$, triés, et dénombrés selon la méthode de comptage de Ložek (1964) (tab. 1). Le volume des échantillons prélevés à Curgies est inférieur de moitié à celui préconisé par Ložek (1964) et Puisségur (1976) pour les lœss. Les valeurs d'abondance obtenues pour ce site n'ont toutefois pas été doublées pour conserver l'intégrité des données de comptage. La composition des assemblages ne montre en outre aucun changement aberrant suggérant une mauvaise représentativité de la malacofaune dans ces échantillons. Les exigences écologiques des taxons ont été compilées d'après divers ouvrages de référence (tab. 2). Pour l'interprétation des données malacologiques, nous avons aussi utilisé les spectres écologiques de Ložek (1964) et de Puisségur (1976), et les indices de diversité et d'équitabilité de Simpson (1949) et de Shannon (Shannon \& Weaver, 1949). Le rapport Juvéniles/Adultes (J/A) proposé par Moine et al. (2005) a été calculé pour les populations des taxons du genre Pupilla. Les courbes obtenues pour P. muscorum et $P$. alpicola montrant les mêmes variations, leurs effec- tifs ont été fusionnés pour le calcul de cet indice. En théorie, lors d'une période climatique plus favorable, l'abondance augmente indépendamment de la proportion en individus juvéniles via le renforcement du cycle de reproduction. Or, en période glaciaire, des valeurs très élevées du rapport $\mathrm{J} / \mathrm{A}$ peuvent accompagner les augmentations d'abondance. La mortalité constatée de nombreux individus juvéniles nés lors des périodes d'amélioration climatique rapides intra-glaciaires peut s'expliquer par une plus grande proportion d'éclosions trop tardives ou trop précoces durant la saison estivale. Les conséquences en sont respectivement une maturité physiologique insuffisante pour permettre l'entrée en hibernation (Moine et al., 2005) ou un effet létal de périodes de gel printanier tardives postérieures au début de la période d'activité printanières des individus juvéniles.

\section{4 - MALACO-BIOGÉOGRAPHIE}

La composition relativement pauvre en malacofaunes des profils de Curgies et de Bourlon (tab. 1) confirme leur appartenance au domaine malaco-biogéographique « ouest» au Pléniglaciaire supérieur (fig. 2). L'enregistrement malacologique de Curgies est le premier à inclure la totalité des taxons caractérisant le domaine «ouest» dans plusieurs échantillons d'une même séquence. Cette caractéristique peut être due à la proximité de la limite avec le domaine «central» où la malacofaune est plus diversifiée, ou à des conditions micro-environnementales favorables à la diversification de la malacofaune. En outre, on notera la présence dans les deux sites de Pupilla alpicola, identifiée pour la première fois dans des dépôts lœssiques du Pléniglaciaire supérieur du nord de la France (fig. 5). Cette espèce caractéristique des environnements palustres (tab. 2) est parfois difficile à différencier de Pupilla muscorum qui est plutôt une espèce polyvalente de milieux ouverts secs ou humides. En raison de la grande variabilité morphologique de

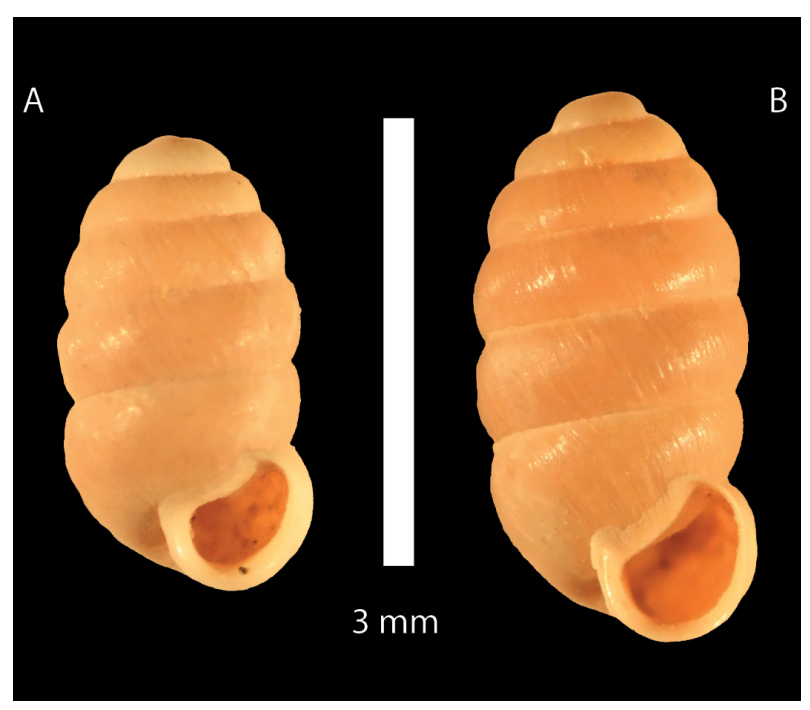

Fig. 5 : Coquilles typiques de Pupilla muscorum (A) et Pupilla alpicola (B) provenant de l'échantillon ZP8-3 de l'enregistrement malacologique de Bourlon.

Fig. 5: Typical shells of Pupilla muscorum (A) and Pupilla alpicola (B) from sample ZP8-3 of Bourlon molluscan record. 
CURGIES

\begin{tabular}{|c|c|c|c|c|c|c|c|c|c|c|c|c|}
\hline $\begin{array}{l}\text { Echan- } \\
\text { tillons }\end{array}$ & $\begin{array}{c}\text { Columella } \\
\text { columella } \\
\text { (5) }\end{array}$ & $\begin{array}{c}\text { Pupilla } \\
\text { muscorum } \\
\text { (5) }\end{array}$ & Limaces ( $\left.7^{\prime}\right)$ & $\begin{array}{l}\text { Trochulus } \\
\text { hispidus (7) }\end{array}$ & $\begin{array}{l}\text { Succinella } \\
\text { oblonga (8) }\end{array}$ & $\begin{array}{c}\text { Pupilla } \\
\text { alpicola (9) }\end{array}$ & $\begin{array}{c}\text { Oxyloma } \\
\text { elegans (9) }\end{array}$ & $\begin{array}{l}\text { Pupilla sp. } \\
\text { (Indet.) }\end{array}$ & $\begin{array}{l}\text { Indéter- } \\
\text { minés }\end{array}$ & Abondance & Richesse & $\begin{array}{c}\text { Zonation } \\
\text { malaco- } \\
\text { logique }\end{array}$ \\
\hline 10 & 0 & 1 & 0 & 0 & 0 & 0 & 5 & 0 & 0 & 6 & 2 & \multirow{3}{*}{ MZ4 } \\
\hline 9 & 0 & 0 & 0 & 0 & 1 & 0 & 4 & 1 & 0 & 6 & 3 & \\
\hline 8 & 0 & 0 & 0 & 1 & 19 & 0 & 3 & 0 & 0 & 23 & 3 & \\
\hline 7 & 0 & 23 & 2 & 0 & 224 & 9 & 83 & 0 & 0 & 341 & 5 & \multirow{2}{*}{ MZ3 } \\
\hline 6 & 1 & 35 & 4 & 1 & 73 & 9 & 61 & 0 & 0 & 184 & 7 & \\
\hline 5 & 2 & 33 & 14 & 7 & 39 & 9 & 3 & 0 & 0 & 107 & 7 & \multirow{2}{*}{ MZ2 } \\
\hline 4 & 0 & 24 & 9 & 4 & 13 & 9 & 0 & 0 & 0 & 59 & 5 & \\
\hline 3 & 0 & 0 & 0 & 1 & 0 & 0 & 0 & 0 & 1 & 2 & 2 & \multirow{3}{*}{ MZ1 } \\
\hline 2 & 0 & 0 & 0 & 0 & 0 & 0 & 0 & 0 & 0 & 0 & 0 & \\
\hline 1 & 0 & 0 & 0 & 0 & 0 & 0 & 0 & 0 & 0 & 0 & 0 & \\
\hline $\begin{array}{l}\text { Echan- } \\
\text { tillons }\end{array}$ & $\begin{array}{c}\text { Columella } \\
\text { columella (5) }\end{array}$ & $\begin{array}{c}\text { Pupilla } \\
\text { muscorum } \\
\text { (5) }\end{array}$ & Limaces (7') & $\begin{array}{c}\text { Trochulus } \\
\text { hispidus (7) }\end{array}$ & $\begin{array}{c}\text { Succinea } \\
\text { oblonga (8) }\end{array}$ & $\begin{array}{c}\text { Pupilla } \\
\text { alpicola (9) }\end{array}$ & $\begin{array}{c}\text { Oxyloma } \\
\text { elegans (9) }\end{array}$ & $\begin{array}{l}\text { Pupilla sp. } \\
\text { (Indet.) }\end{array}$ & $\begin{array}{c}\text { Indéter- } \\
\text { minés }\end{array}$ & Abondance & Richesse & $\begin{array}{c}\text { Zonation } \\
\text { malaco- } \\
\text { logique }\end{array}$ \\
\hline 10 & 0,00 & 16,67 & 0,00 & 0,00 & 0,00 & 0,00 & 83,33 & 0,00 & 0,00 & 6 & 2 & \multirow{3}{*}{ MZ4 } \\
\hline 9 & 0,00 & 0,00 & 0,00 & 0,00 & 16,67 & 0,00 & 66,67 & 16,67 & 0,00 & 6 & 3 & \\
\hline 8 & 0,00 & 0,00 & 0,00 & 4,35 & 82,61 & 0,00 & 13,04 & 0,00 & 0,00 & 23 & 3 & \\
\hline 7 & 0,00 & 6,74 & 0,59 & 0,00 & 65,69 & 2,64 & 24,34 & 0,00 & 0,00 & 341 & 5 & \multirow{2}{*}{ MZ3 } \\
\hline 6 & 0,54 & 19,02 & 2,17 & 0,54 & 39,67 & 4,89 & 33,15 & 0,00 & 0,00 & 184 & 7 & \\
\hline 5 & 1,87 & 30,84 & 13,08 & 6,54 & 36,45 & 8,41 & 2,80 & 0,00 & 0,00 & 107 & 7 & \multirow{2}{*}{ MZ2 } \\
\hline 4 & 0,00 & 40,68 & 15,25 & 6,78 & 22,03 & 15,25 & 0,00 & 0,00 & 0,00 & 59 & 5 & \\
\hline 3 & 0,00 & 0,00 & 0,00 & 50,00 & 0,00 & 0,00 & 0,00 & 0,00 & 50,00 & 2 & 2 & \multirow{3}{*}{ MZ1 } \\
\hline 2 & 0,00 & 0,00 & 0,00 & 0,00 & 0,00 & 0,00 & 0,00 & 0,00 & 0,00 & 0 & 0 & \\
\hline 1 & 0,00 & 0,00 & 0,00 & 0,00 & 0,00 & 0,00 & 0,00 & 0,00 & 0,00 & 0 & 0 & \\
\hline
\end{tabular}

BOURLON

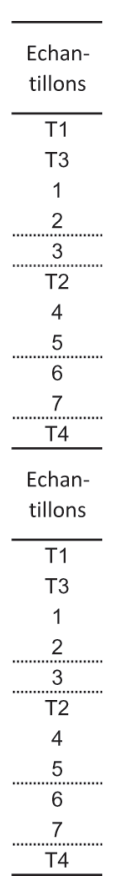

\begin{tabular}{|c|c|c|c|c|}
\hline $\begin{array}{l}\text { Pupilla } \\
\text { muscorum } \\
\text { (5) }\end{array}$ & Limaces $\left(7^{\prime}\right)$ & $\begin{array}{l}\text { Trochulus } \\
\text { hispidus (7) }\end{array}$ & $\begin{array}{l}\text { Succinella } \\
\text { oblonga (8) }\end{array}$ & $\begin{array}{c}\text { Pupilla } \\
\text { alpicola (9) }\end{array}$ \\
\hline 20 & 8 & 0 & 2 & 0 \\
\hline 10 & 6 & 1 & 6 & 0 \\
\hline 5 & 1 & 1 & 1 & 0 \\
\hline 13 & 7 & 0 & 7 & 6 \\
\hline 322 & 39 & 0 & 232 & 162 \\
\hline 748 & 239 & 1 & 236 & 20 \\
\hline 424 & 140 & 0 & 310 & 41 \\
\hline 515 & 181 & 0 & 479 & 65 \\
\hline 950 & 321 & 0 & 696 & 100 \\
\hline 922 & 281 & 0 & 364 & 120 \\
\hline 59 & 12 & 0 & 1 & 0 \\
\hline $\begin{array}{l}\text { Pupilla } \\
\text { muscorum } \\
\text { (5) }\end{array}$ & Limaces ( $\left(7^{\prime}\right)$ & $\begin{array}{l}\text { Trochulus } \\
\text { hispidus (7) }\end{array}$ & $\begin{array}{l}\text { Succinella } \\
\text { oblonga (8) }\end{array}$ & $\begin{array}{c}\text { Pupilla } \\
\text { alpicola (9) }\end{array}$ \\
\hline 66,67 & 26,67 & 0,00 & 6,67 & 0,00 \\
\hline 43,48 & 26,09 & 4,35 & 26,09 & 0,00 \\
\hline 62,50 & 12,50 & 12,50 & 12,50 & 0,00 \\
\hline 39,39 & 21,21 & 0,00 & 21,21 & 18,18 \\
\hline 42,65 & 5,17 & 0,00 & 30,73 & 21,46 \\
\hline 60,13 & 19,21 & 0,08 & 18,97 & 1,61 \\
\hline 46,34 & 15,30 & 0,00 & 33,88 & 4,48 \\
\hline 41,53 & 14,60 & 0,00 & 38,63 & 5,24 \\
\hline 45,96 & 15,53 & 0,00 & 33,67 & 4,84 \\
\hline 54,65 & 16,66 & 0,00 & 21,58 & 7,11 \\
\hline 81,94 & 16,67 & 0,00 & 1,39 & 0,00 \\
\hline
\end{tabular}

\begin{tabular}{|c|c|c|}
\hline Abondance & Richesse & $\begin{array}{c}\text { Zonation } \\
\text { malaco- } \\
\text { logique }\end{array}$ \\
\hline 30 & 3 & \multirow{4}{*}{ MZ4 } \\
\hline 23 & 4 & \\
\hline 8 & 4 & \\
\hline 33 & 4 & \\
\hline 755 & 4 & $M Z 3 b$ \\
\hline 1244 & 5 & \multirow{3}{*}{ MZ3a } \\
\hline 915 & 4 & \\
\hline 1240 & 4 & \\
\hline 2067 & 4 & \multirow{2}{*}{ MZ2 } \\
\hline 1687 & 4 & \\
\hline 72 & 3 & MZ1 \\
\hline Abondance & Richesse & $\begin{array}{c}\text { Zonation } \\
\text { malaco- } \\
\text { logique }\end{array}$ \\
\hline 30 & 3 & \multirow{4}{*}{ MZ4 } \\
\hline 23 & 4 & \\
\hline 8 & 4 & \\
\hline 33 & 4 & \\
\hline 755 & 4 & $M Z 3 b$ \\
\hline 1244 & 5 & \multirow{3}{*}{ MZ3a } \\
\hline 915 & 4 & \\
\hline 1240 & 4 & \\
\hline 2067 & 4 & \multirow{2}{*}{ MZ2 } \\
\hline 1687 & 4 & \\
\hline 72 & 3 & MZ1 \\
\hline
\end{tabular}

Tab. 1 : Comptages bruts et pourcentages des taxons identifiés dans les assemblages malacologiques du Pléniglaciaire supérieur weichselien de Curgies et de Bourlon.

Les espèces sont ordonnées par groupes écologiques (cf. tab. 2)

Tab. 1: Absolute and relative frequencies of taxa identified in Weichselian Upper Pleniglacial molluscan assemblages from Curgies and Bourlon. Species are ordinated by ecological groups (cf. tab. 2).

Pupilla muscorum résultant de sa plasticité écophénotypique (Rousseau \& Laurin, 1984; Rousseau, 1997), ses morphotypes de milieux très froids et très humides à la spire haute et à l'ouverture arrondie et peu épaissie peuvent être très semblables à ceux de Pupilla alpicola. Une révision du matériel malacologique du domaine «ouest» en grande partie antérieur à ces analyses morphométriques permettrait de rechercher la présence de Pupilla alpicola et donc l'occurrence de phases palustres dans les séquences lœssiques concernées.

\section{4 - RÉSULTATS}

\section{1 - DÉFINITION DES MALACOZONES DE CURGIES ET DE BOURLON}

Les deux enregistrements malacologiques (fig. 6 et 7 , tab. 1) ont été chacun divisés en plusieurs malacozones sur la base des combinaisons de variations les plus significatives et les plus diagnostiques de différents paramètres des malacofaunes (tab. 3). 


\begin{tabular}{|c|c|c|c|c|}
\hline Curgies & Bourlon & Taxons & Groupes écologiques & Habitat \\
\hline $\mathrm{x}$ & - & $\begin{array}{l}\text { Columella columella (G. } \\
\text { von Martens, 1830) }\end{array}$ & 5: Milieu ouvert & $\begin{array}{l}\text { Régions froides et stations détrempées avec végétation (marécages herbeux, forêts } \\
\text { subarctiques et prairies) }\end{array}$ \\
\hline$x$ & $x$ & $\begin{array}{l}\text { Pupilla muscorum } \\
\text { (Linnaeus, 1758) }\end{array}$ & 5: Milieu ouvert & Stations ouvertes, ensoleillées, et sèches ou humides \\
\hline $\mathrm{x}$ & $\mathrm{x}$ & $\begin{array}{l}\text { Trochulus hispidus } \\
\text { (Linnaeus, 1758) }\end{array}$ & 7: Mésophiles & $\begin{array}{l}\text { Dans tous types de stations, excepté les plus sèches; nécessite un couvert végétal plus } \\
\text { développé que Pupilla muscorum en période glaciaire }\end{array}$ \\
\hline $\mathrm{x}$ & $x$ & Limaces & 7': Limaces & $\begin{array}{l}\text { Variable en fonction des taxons; la limacelle (coquille interne) ne permet toutefois pas } \\
\text { I'identification spécifique des taxons qui en sont pourvus }\end{array}$ \\
\hline $\mathrm{x}$ & $\mathrm{x}$ & $\begin{array}{l}\text { Succinella oblonga } \\
\text { (Draparnaud, 1801) }\end{array}$ & 8: Hygrophiles & $\begin{array}{c}\text { Stations détrempées avec une végétation clairsemées (prairies humides, marécages et à } \\
\text { proximité de ruisseaux) }\end{array}$ \\
\hline $\mathrm{x}$ & $\mathrm{x}$ & $\begin{array}{l}\text { Pupilla alpicola } \\
\text { (Charpentier, 1837) }\end{array}$ & 9: Palustres & $\begin{array}{c}\text { Stations de montagne détrempées (marécages, tourbières, prairies détrempées) de } \\
\text { préférence dans les zones calcaires }\end{array}$ \\
\hline $\mathrm{x}$ & - & $\begin{array}{l}\text { Oxyloma elegans (Risso, } \\
\text { 1826) }\end{array}$ & 9: Palustres & $\begin{array}{l}\text { Milieux palustres (marécages, tourbières, bords de rivières,...) directement au contact de } \\
\text { l'eau sur le sol ou les plantes; stations de basse altitude }\end{array}$ \\
\hline
\end{tabular}

Tab. 2: Caractérisation écologique des taxons malacologiques identifiés dans les dépôts lœssiques du Pléniglaciaire supérieur weichselien de Curgies et Bourlon.

La taxonomie a été établie d'après Anderson (2005), Bank et al. (2001), Falkner et al. (2002) and Rocroi et al. (2007). Les exigences écologiques des taxons ont été compilées d'après Adam (1960), Germain (1930), Kerney et al. (1983), Likhachev et Rammel'meier (1962), Ložek (1964), Puisségur (1976) et Zilch et Jaeckel (1962).

Tab. 2: Ecological characteristics of molluscan taxa identified in Weichselian Upper Pleniglacial loessic deposits from Curgies and Bourlon. The taxonomy is based on Anderson (2005), Bank et al. (2001), Falkner et al. (2002), and Rocroi et al., 2007. Ecological requirements have been compiled from Adam (1960), Germain (1930), Kerney et al. (1983), Likhachev and Rammel'meier (1962), Ložek (1964), Puisségur (1976) and Zilch and Jaeckel (1962).

\begin{tabular}{|c|c|c|c|c|c|c|c|c|c|c|c|c|c|c|c|}
\hline SITES & & MALACOZONES & 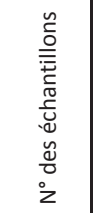 & 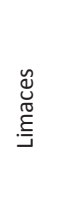 & 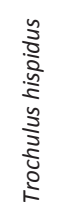 & 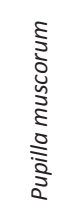 & 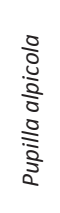 & 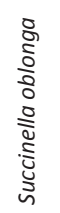 & 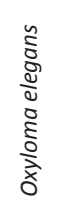 & 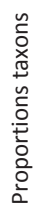 & 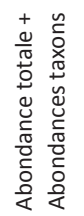 & 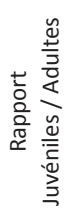 & 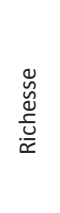 & 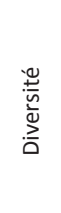 & 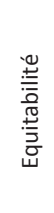 \\
\hline \multirow{4}{*}{$\begin{array}{l}\mathrm{C} \\
U \\
\mathrm{R} \\
\mathrm{G} \\
\mathrm{I} \\
\mathrm{E} \\
\mathrm{S}\end{array}$} & MZ4 & Zone à $O$. elegans & $8-10$ & \multirow{4}{*}{---} & \multirow{4}{*}{---} & & & +++ & +++ & & ע & & $\checkmark$ & $\rightarrow$ & $\pi$ \\
\hline & MZ3 & $\begin{array}{c}\text { Zone à } S \text {. oblonga et } O \text {. } \\
\text { elegans }\end{array}$ & $6-7$ & & & & & \multirow[t]{3}{*}{ ス } & ス & & ス & $\operatorname{Max}$ & עע & עע & עע \\
\hline & MZ2 & $\begin{array}{l}\text { Zone à } T \text {. hispidus et } \\
\text { limaces }\end{array}$ & $4-5$ & & & & & & Min & & $\pi$ & $\operatorname{Min}$ & $\pi$ & $\operatorname{Max}$ & \\
\hline & MZ1 & & $1-3$ & & & & & & & & $\sim 0$ & & & & \\
\hline \multirow{14}{*}{$\begin{array}{l}\text { B } \\
O \\
U \\
R \\
L \\
O \\
N\end{array}$} & MZ5 & Zone à $P$. muscorum & $\begin{array}{c}2-1+T 3 \\
+T 1\end{array}$ & \multirow{5}{*}{ \ } & & $\begin{array}{c}\%> \\
\% \text { SOBL }\end{array}$ & --- & & & \multirow{5}{*}{$\rightarrow$} & $\sim 0$ & $<0$ & \multirow{5}{*}{$\rightarrow$} & \multirow{4}{*}{$\rightarrow$} & \multirow{4}{*}{$\rightarrow$} \\
\hline & MZ4 & Zone à $P$. alpicola & 3 & & & $\searrow \searrow$ & スス & & & & 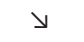 & $\pi \pi$ & & & \\
\hline & MZ3 & $\begin{array}{c}\text { Zone à S. oblonga et } P \text {. } \\
\text { muscorum }\end{array}$ & $5-4+T 2$ & & & & & & & & עע & $\pi$ & & & \\
\hline & MZ2 & $\begin{array}{l}\text { Zone à } P \text {. muscorum et } \\
\text { S. oblonga }\end{array}$ & $7-6$ & & & & & ス & & & $\begin{array}{c}\operatorname{Max}+ \\
\pi\end{array}$ & $\sim 0$ & & & \\
\hline & MZ1 & Zone à $P$. muscorum & T4 & & & +++ & & --- & & & $\pi$ & $\sim 0$ & & Min & Min \\
\hline & $\pi$ & Faible augmentation & & & & & & & & & & & & & \\
\hline & ת & Forte augmentation & & & & & & & & & & & & & \\
\hline & y & Faible diminution & & & & & & & & & & & & & \\
\hline & $\searrow \searrow$ & Forte diminution & & & & & & & & & & & & & \\
\hline & $\rightarrow$ & Stagnation & & & & & & & & & & & & & \\
\hline & +++ & Prédominance & & & & & & & & & & & & & \\
\hline & --- & Quasi-absence & & & & & & & & & & & & & \\
\hline & $\%$ & Proportions & & & & & & & & & & & & & \\
\hline & $\sim 0$ & Valeur très faible & & & & & & & & & & & & & \\
\hline
\end{tabular}

Tab. 3 : Critères de définition des malacozones individualisées dans les séquences lœssiques de Curgies et de Bourlon.

$(\boldsymbol{\lambda})$ faible augmentation, $(\boldsymbol{\lambda})$ forte augmentation, $(\boldsymbol{V})$ faible diminution, $(\boldsymbol{V})$ forte diminution, $(\rightarrow)$ stagnation, $(+++)$ prédominance, $(---)$ quasiabsence, $(\%)$ proportions, $(\sim 0)$ valeurs très faibles.

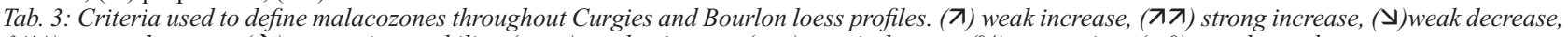
$(\mathrm{\searrow})$ strong decrease, $(\rightarrow)$ stagnation, stability, (+++) predominance, (- - ) quasi-absence, (\%) proportion, $(\sim 0)$ very low values. 


\section{2 - INTERPRÉTATIONS PALÉOENVIRONNEMEN- TALES DE LA SÉQUENCE DE CURGIES}

MZ1 «Zone à $\boldsymbol{T}$. hispidus»: une aridité importante et un couvert végétal très peu développé (très faible nombre de granules de vers de terre) peuvent expliquer la quasiabsence de mollusques terrestres et la faible épaisseur des deux gleys de toundra. Aucun indice ne suggère que les coquilles aient pu être détruites postérieurement à leur enfouissement.

MZ2 «Zone à T. hispidus et limaces» : dans la moitié inférieure du gley de toundra, les proportions des taxons et des spectres écologiques sont relativement équilibrées, et suggèrent la présence d'une micro-mosaïque environnementale ouverte, très humide et peu végétalisée. Le milieu devient plus humide (proportion croissante du taxon hygrophile Succinella oblonga et apparition en faibles effectifs du taxon palustre Oxyloma elegans et de Columella columella, espèce typique des stations de montagne humides à couvert végétal), mais les températures notamment estivales restent froides (apparition du taxon boréo-alpin Columella columella, et valeurs faibles du rapport $\mathrm{J} / \mathrm{A}$ et de l'abondance). Ces conditions ont conduit à la formation de grandes fentes en coin en liaison avec un pergélisol riche en glace.

MZ3 «Zone à S. oblonga et O. elegans»: dans la moitié supérieure du gley de toundra, l'humidification du milieu s'accélère au détriment de la végétation (doublement des effectifs de l'espèce hygrophile Succinella oblonga, disparition des limaces, de Columella columella et de Trochulus hispidus, taxons nécessitant un couvert végétal plus développé), mais la micromosaïque environnementale perdure (maintien simultané de Pupilla muscorum et des taxons palustres Pupilla alpicola et Oxyloma elegans), soit dans l'espace avec des flaques d'eau éparses persistantes alternant avec des points hauts plus secs, soit dans le temps avec une succession saisonnière d'environnements humides puis secs permettant le développement successif des populations des espèces présentes. L'occurrence d'une succession environnementale temporelle a notamment été suggérée par Davies (1996) pour expliquer la présence

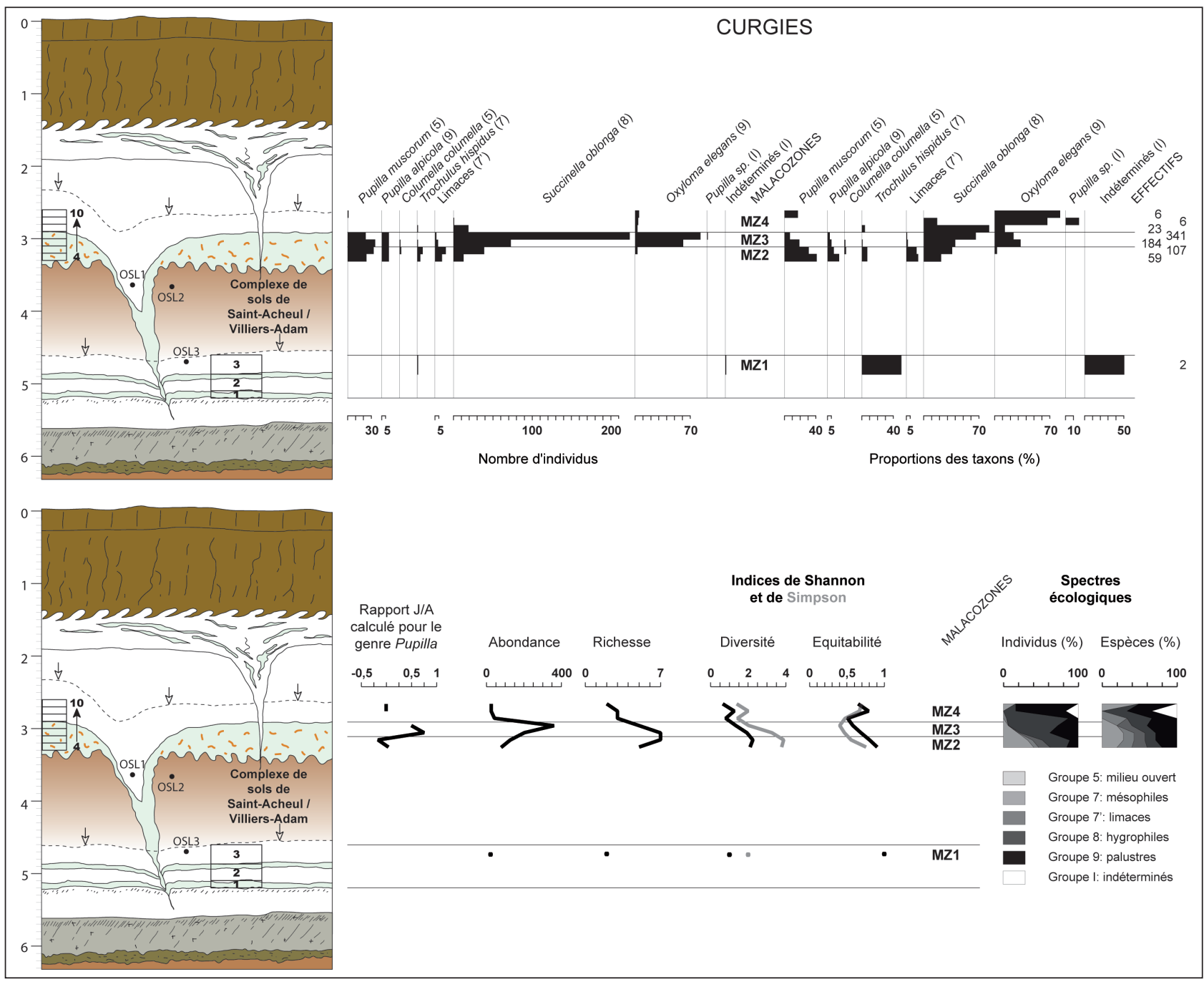

Fig. 6 : Profil stratigraphique, diagrammes malacologiques et indices écologiques des malacofaunes du site de Curgies.

Les échantillons malacologiques sont numérotés 1 à 3 dans la colonne inférieure et 4 à 10 dans la colonne supérieure. Voir le tableau 2 pour les caractéristiques écologiques des taxons.

Fig. 6: Stratigraphical profile, malacological diagrams and ecological indexes of malacofaunas for the site of Curgies. Molluscan samples are numbered 1 to 3 in the lower column and 4 to 10 in the upper column. See table 2 for ecological characteristics of taxa. 
de Pupilla muscorum au sein d'assemblages malacologiques dominés par des cortèges d'espèces aquatiques, palustres et de milieux humides dans des dépôts alluviaux holocènes. Au sommet du gley de toundra, l'humidité est maximale et la végétation très appauvrie: on observe une stagnation des effectifs de P. muscorum et $P$. alpicola, et une explosion démographique des populations hygrophiles et palustres de $S$. oblonga et $O$. elegans. Par ailleurs l'augmentation importante du rapport J/A et des abondances de $S$. oblonga et $O$. elegans indique un radoucissement sensible des températures. Dans le cas de Nussloch, la position de la séquence en sommet de greda (ride de lœss allongée) a favorisé le drainage des eaux de fonte, permettant à tous les taxons présents d'accroître leur population sous l'effet de l'amélioration climatique. A Curgies, un environnement très humide résulte du mauvais drainage des eaux de fonte, et l'intensification du cycle de reproduction ne profite qu'aux taxons hygrophiles et palustres se contentant d'un couvert végétal pauvre. Une fois passé l'optimum thermique, les phases de regel hivernal provoquent la cryoturbation du gley de toundra par mise en pression de la couche active entre la table du pergélisol et la surface à nouveau gelée du sol (Van Vliet-Lanoë, 1985, 1987).

MZ4 «Zone à O. elegans» : cette dernière malacozone est caractérisée par le retour brutal d'une sédimentation lœssique typique, par la fin des processus de cryoturbation du gley de toundra, et par des températures estivales froides (chute drastique de l'abondance de tous les taxons). Les transitions gley-loess s'accompagnent aussi généralement d'un milieu plus sec (augmentation des proportions en taxons de milieu ouvert et en individus mésophiles). Dans le cas présent, le milieu reste très humide et peu favorable à la diversification végétale (maintien de l'espèce palustre Oxyloma elegans, disparition de l'espèce palustre Pupilla alpicola, disparition progressive de Succinella oblonga, présence sporadique de Pupilla muscorum et Trochulus hispidus, et richesse et diversité minimales), probablement en raison d'une dépression générée par la fonte du coin de glace qui a favorisé le maintien d'eau à cet endroit. Cette situation n'est pas unique, il arrive parfois de trouver des taxons aquatiques d'eau douce en grande quantité dans des sédiments lœssiques (Mazenot, 1951; Moine et al., 2005) qui témoignent d'environnements inondés plusieurs mois par an. Des malacofaunes similaires, bien qu'un peu plus riches, ont été observées dans les dépôts du Dryas Récent du nord de la France et ont conduit à une interprétation similaire (Limondin \& Antoine, 2001).

Les périodes de transition gley-lœss sont souvent mal enregistrées dans les sédiments en raison de leur courte durée et des perturbations stratigraphiques générées par les phénomènes de cryoturbation (présence d'une zone de mélange de 15 à 20 centimètres d'épaisseur). En outre, leur caractérisation précise nécessiterait de séparer les loess piégés entre les involutions des gleys de toundra dans les zones cryoturbées, ce qui est irréalisable compte tenu du volume de prélèvement nécessaire aux analyses malacologiques. De même qu'à Nussloch, cette transition semble plus rapide que la transition inverse loss-gley.

\section{3 - INTERPRÉTATIONS PALÉOENVIRONNEMEN- TALES DE LA SÉQUENCE DE BOURLON}

MZ1 «Zone à P. muscorum»: le complexe de sols de Saint-Acheul / Villiers-Adam semble caractérisé par des conditions environnementales sèches (prédominance nette de l'espèce de milieu ouvert Pupilla muscorum), une végétation peu diversifiée (pauvreté de la malacofaune) et des températures très froides (abondances faibles). Ces conditions environnementales peuvent toutefois caractériser la période de mise en place du dépôt loessique plutôt que sa transformation ultérieure en limon brun. Le sommet du complexe de sols de Saint-Acheul / Villiers-Adam n'est donc pas toujours totalement décalcifié dans le nord de la France. De nouveaux échantillonnages en colonne continue permettront de mieux connaître la composition des malacofaunes et l'évolution paléoenvironnementale au Pléniglaciaire moyen weichselien dans cette région.

MZ2 «Zone à $\boldsymbol{P}$. muscorum et $\boldsymbol{S}$. oblonga»: un réchauffement important intervient à la base (apparente) du gley de toundra (abondances maximales de la majorité des taxons, valeurs constantes et peu élevées du rapport $\mathrm{J} / \mathrm{A}$ ). La mosaïque environnementale est aussi humide qu'en surface d'un gley de toundra (augmentation des proportions de l'espèce hygrophile Succinella oblonga).

MZ3 «Zone à S. oblonga et P. muscorum» : les températures diminuent mais restent douces (forte baisse des effectifs qui restent néanmoins élevés dans ce contexte, valeurs toujours constantes du rapport J/A). Le milieu est un peu moins humide (légère baisse des proportions en taxons hygrophiles et palustres). Cette phase post-réchauffement est plutôt longue et bien enregistrée contrairement à celle associée à la transition gley-lœss à Curgies. Les proportions légèrement plus élevées de Pupilla muscorum et des limaces au détriment de celles de Succinella oblonga dans l'échantillon T2 par rapport aux échantillons 5 et 4 suggèrent des conditions un peu moins humides peut-être dues à une faible surélévation ou au drainage des eaux de surface aux abords du coin de glace en activité.

MZ4 «Zone à P. alpicola»: un second réchauffement, moins intense, intervient au sommet du gley de toundra (accroissement soudain de la population de l'espèce palustre Pupilla alpicola). Contrairement à celui de MZ2, il se caractérise par un allongement de la saison de reproduction avec persistance d'hivers toujours rigoureux et/ ou de gelées printanières intenses (forte augmentation $\mathrm{du}$ rapport $\mathrm{J} / \mathrm{A}$ indiquant une surmortalité printanière ou automnale importante des juvéniles). L'humidité augmente à nouveau (baisse accrue des effectifs de Pupilla muscorum et des limaces, et arrêt de la baisse des effectifs de Succinella oblonga plus hygrophile) probablement en raison de la fonte saisonnière plus prononcée du pergélisol, ayant également entraîné la cryoturbation du gley de toundra.

Cet épais gley de toundra pourrait-il être biphasé? Cette situation a déjà été observée auparavant. D'une part, dans la séquence de lœss de Savy (Aisne, France), un gley de toundra, situé dans la même position stratigraphique que celui de Bourlon, apparaît constitué de deux unités distinctes séparées par un niveau de pseudomor- 


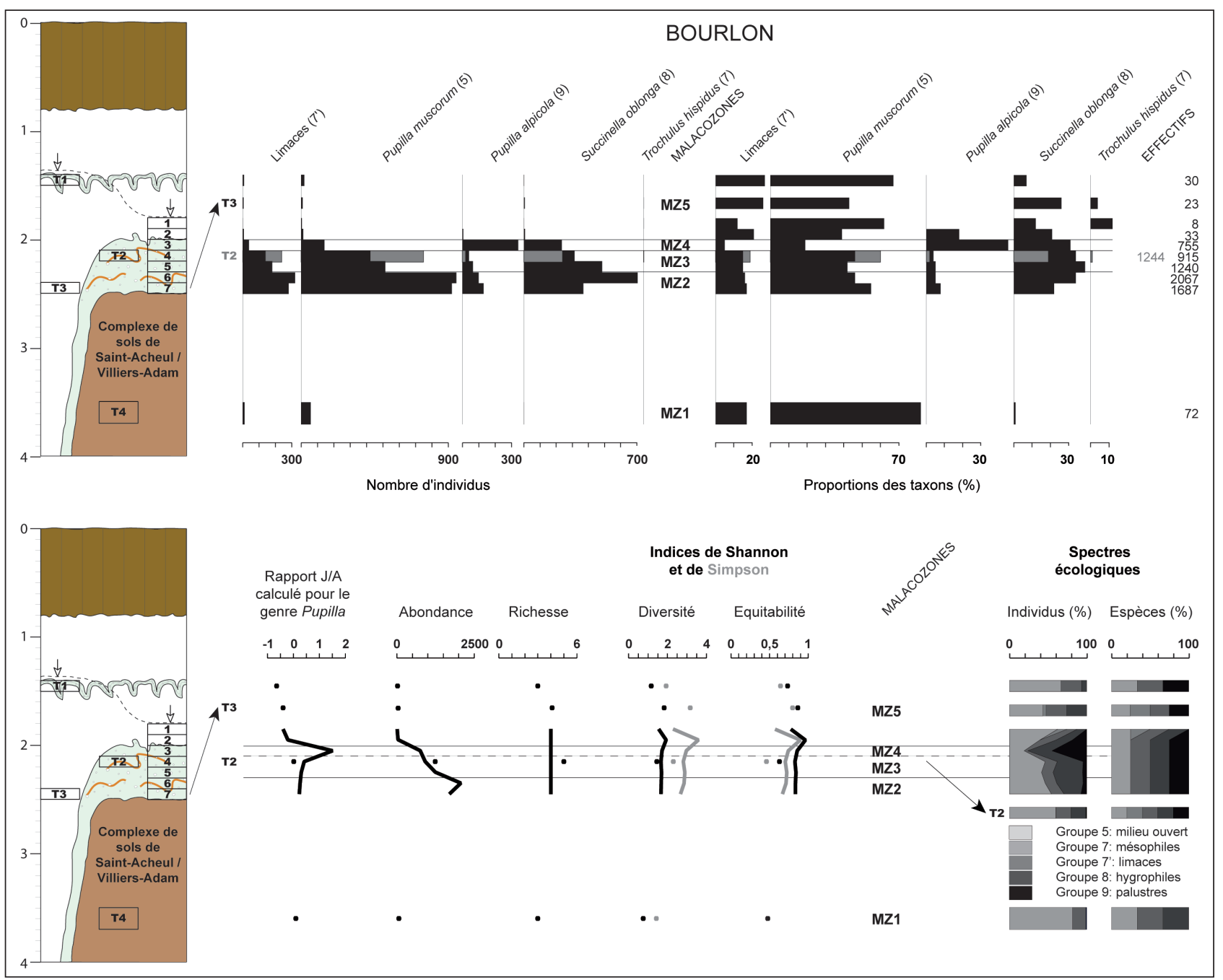

Fig. 7 : Profil stratigraphique, diagrammes malacologiques et indices écologiques des malacofaunes du site de Bourlon.

Les échantillons malacologiques sont numérotés 1 à 7 dans la colonne continue et T1 à T4 pour les échantillons-tests isolés. Voir le tableau 2 pour les caractéristiques écologiques des taxons.

Fig. 7: Stratigraphical profile, malacological diagrams and ecological indexes of malacofaunas for the site of Bourlon. Molluscan samples are numbered from 1 to 7 in the continuous column and T1 to T4 for isolated test-samples. See table 2 for ecological characteristics of taxa.

phoses de grandes fentes à coin de glace colmatées par des lœss homogènes (Locht et al., 2006). D'autre part, dans la séquence de lœss de Nussloch, un épais gley de toundra (G2) observé dans les premiers profils analysés (P2 et P3) (Antoine et al., 2001) s'est finalement révélé être constitué de deux unités distinctes (G2a et G2b) dans le dernier profil (P4) mis au jour (Antoine et al., 2002). La surimposition de deux gleys de toundra distincts à Bourlon est donc tout à fait plausible dans un contexte local à faible taux de sédimentation.

MZ5 «Zone à $P$. muscorum»: la reprise de la sédimentation lœssique s'accompagne de conditions beaucoup plus froides (réduction drastique des populations, rapport J/A très faible), plus sèches (disparition de l'espèce palustre Pupilla alpicola et faibles proportions en taxons hygrophiles) et d'une légère diversification végétale (réapparition sporadique de Trochulus hispidus). Le coin de glace ayant fondu, la grande fente a pu localement piéger ou retenir plus d'humidité ou d'eau expliquant ainsi les $26 \%$ de Succinella oblonga de l'assemblage T3 dans ce contexte lossique. Le matériel malacologique du genre Pupilla de cette unité loessique se caractérise par un déficit en apex, qui est la partie la plus fine, légère et fragile de la coquille (valeurs de plus en plus négatives du rapport Juvéniles/Adultes). La même observation a déjà été faite dans les niveaux les moins abondants des loss de Nussloch (Moine et al., 2008). En l'absence de traces de destruction physique ou chimique sur les coquilles récoltées, ce manque d'apex peut résulter de la très faible concentration en matériel dans les échantillons, ou de pertes durant les phases d'échantillonnage et de tamisage en raison de cette très faible concentration. La composition similaire des échantillons malacologiques 2 et $\mathrm{T} 3$ suggère un remplissage rapide de la grande fente en coin ainsi que leur contemporanéité. Enfin, au niveau de l'échantillon $\mathrm{T} 1$ intervient une augmentation d'humidité (formation du gley de toundra à polygones décimétriques) accompagnée d'un appauvrissement de la végétation (absence de Trochulus hispidus, comme dans l'échantillon de base de cette malacozone). Aucun réchauffement ne semble associé à ce gley de toundra peu épais (absence d'augmentations importantes de l'abondance et du rapport J/A) dont la composition de la malacofaune est plus proche de celle du loess sous-jacent que du gley de toundra épais. 


\section{5 - DISCUSSION}

\section{1 - IMPLICATIONS PALÉOCLIMATIQUES}

La dynamique régionale de formation des gleys de toundra en Europe de l'ouest et le modèle conceptuel établi à Nussloch (voir Contexte et fig. 3) nous permettent, en l'absence de datations radiométriques pour les profils de Curgies et de Bourlon, de proposer des corrélations entre les changements paléoenvironnementaux déduits de l'analyse des malacofaunes et des sédiments, et les différentes phases des cycles stades-interstades de Dansgaard-Oeschger (tab. 4) à l'instar de ce qui a été proposé pour la séquence malacologique de Nussloch.

Dans les deux sites étudiés, seule la séquence de Curgies présente l'enregistrement d'une transition stadeinterstade (Zone à T. hispidus et limaces). A Nussloch, ce type de phase de transition est associé au développement d'un gley de toundra (Moine et al., 2008) par engorgement de la couche active suite à la formation d'une nappe perchée au sommet du pergélisol (Haesaerts \& Van Vliet-Lanoë, 1981). Le gel saisonnier accompagné de la formation de lentilles de glace cisaillant les racines dans le sol aurait conduit à l'appauvrissement de la végétation. Ces phénomènes seraient favorisés par des épisodes d'affaiblissement des hautes pressions de la cellule polaire générant une pénétration plus fréquente des dépressions en provenance de l'Atlantique Nord et une augmentation des précipitations (Hatté \& Guiot, 2005), très probablement sous la forme de neige.

Durant les interstades (MZ3 à Curgies; MZ2 à Bourlon), l'arrivée d'un peu de chaleur d'origine océanique sur le continent provoque une augmentation de l'intensité de la fonte saisonnière du sommet du pergélisol et débouche sur la fusion des coins de glace (approfondissement de la couche active, épaississement de l'horizon de gley dans les dépôts sous-jacents, fluage du gley sur les bords des grandes fentes après la fonte des coins de glace). Cet apport de chaleur est à relier à des périodes d'affaiblissement maximal des hautes pressions de la cellule polaire qui permettent le passage plus fréquent de dépressions atlantiques sur l'Europe de l'ouest. Toutefois, cette tendance s'inverse rapidement, et la fin des phases interstadiaires se caractérise déjà par un début de baisse des températures, notamment estivales, et le ralentissement des processus de cryoturbation et (ou) de gélifluxion des gleys de toundra (Antoine et al., 2009).

A Curgies comme à Bourlon, le brusque retour de la sédimentation lossique et de conditions très froides et sèches au sommet de l'épais gley de toundra central suggèrent un rééquilibrage tout aussi brutal des masses d'air. La brièveté de cette phase de transition interstadestade ne permet pas de lui associer un enregistrement sédimentaire distinct, excepté peut-être les dépôts de lœss piégés entre les involutions qui affectent le sommet des gleys de toundra cryoturbés. Les lœss et les malacofaunes des malacozones supérieures de chaque site sont quant à eux typiques d'une phase stadiaire.

Notons enfin deux événements particuliers. Premièrement, MZ3 est une malacozone particulière présente uniquement à Bourlon. Malgré des effectifs élevés dans ce contexte glaciaire, la forte décroissance des abondances et les faibles valeurs du rapport J/A ne permettent pas d'attribuer cette malacozone à une phase strictement interstadiaire comme MZ2 sous-jacente. Une malacozone aux caractéristiques équivalentes a également été distinguée à Nussloch au sein du gley de toundra G2. Deuxièmement, l'unique échantillon T1 prélevé à Bourlon dans le gley de toundra supérieur à polygones décimétriques ne permet pas d'aborder la dynamique de sa formation. Toutefois, l'absence d'augmentation significative de l'abondance en mollusques qui caractérise ce niveau a déjà été observée dans les gleys de toundra peu épais faiblement ou non cryoturbés de la moitié supérieure des dépôts lœssiques du Pléniglaciaire supérieur de Nussloch, et notamment dans le gley de toundra G8 (équivalent local de l'Horizon à langues de Nagelbeek; Moine et al., 2008). Pourtant, la seule présence des polygones de taille décimétrique et des petites fentes associées, implique au moins une faible augmentation de l'humidité même si la malacofaune ne la reflète pas. Ces phénomènes résulteraient donc d'une phase de transition stade-interstade n'ayant pas débouché sur des conditions climatiques pleinement interstadiaires. Les caractéristiques des gleys de toundra de la seconde moitié des dépôts lœssiques du Pléniglaciaire supérieur de Nussloch (faible épaisseur, quasi-absence de cryoturbations, absence de maxima d'abondance en mollusques), incluant notamment l'équivalent local de l'horizon à langues de Nagelbeek (G8), ont conduit à la même interprétation (Moine et al., 2008).

\section{2 - IMPLICATIONS STRATIGRAPHIQUES}

Les enregistrements malacologiques des gleys de toundra de Curgies et de Bourlon montrent une dynamique différente (aucun indice de bi-partition du gley de toundra à Curgies) (fig. 6 et 7). Malgré leur position stratigraphique similaire, il est probable que leur formation ne soit pas contemporaine et qu'elle résulte donc de cycles climatiques stade-interstade différents. Pour

\begin{tabular}{|c|c|c|c|c|}
\hline \multicolumn{2}{|r|}{ CURGIES - Malacozones } & \multicolumn{2}{|r|}{ BOURLON - Malacozones } & \multirow{2}{*}{$\begin{array}{c}\text { Phases des cycles de D-O } \\
\text { Stade }\end{array}$} \\
\hline MZ4 & Zone à O. elegans & MZ5 & Zone à $P$. muscorum & \\
\hline MZ3 & Zone à S. oblonga et O. elegans & MZ4 & Zone à $P$. alpicola & Interstade \\
\hline \multirow[t]{2}{*}{ MZ2 } & Zone à $T$. hispidus et limaces & $\mathrm{MZ3}$ & Zone à $S$. oblonga et $P$. muscorum & Transition interstade-stade \\
\hline & & MZ2 & Zone à $P$. muscorum et S. oblonga & Interstade \\
\hline
\end{tabular}

Tab. 4 : Mise en parallèle des malacozones définies à Curgies et à Bourlon avec les phases des cycles climatiques millénaires de DansgaardOeschger.

Tab. 4: Parallelism between molluscan zones defined in Curgies and Bourlon with the phases of Dansgaard-Oeschger millennial-timescale climatic cycles. 
tester cette hypothèse, nous les avons comparés avec les enregistrements malacologiques des gleys de toundra de Nussloch en adaptant l'épaisseur des pics d'abondance de Curgies et de Bourlon à ceux de Nussloch (fig. 8).

La structure bi-phasée de l'enregistrement malacologique du gley de toundra de Bourlon permettait d'envisager une corrélation soit avec le gley de toundra G2, soit avec les gleys de toundra G3 et G4 de Nussloch. Après comparaison des données, la première possibilité s'est révélée la plus cohérente (fig. 8) L'analyse malacologique du gley de toundra G2 a été réalisée dans le profil P3 de Nussloch où les subdivisions stratigraphiques de cet horizon n'étaient pas lisibles (Moine et al., 2008). La comparaison des enregistrements malacologiques des gleys de toundra de Nussloch (G2) et de Bourlon montre un profil d'abondance similaire: maxima à la base, décroissance importante et palier au sommet. Dans les deux sites, le second réchauffement apparaît de moindre intensité, et bénéficie surtout au taxon hygrophile Succinella oblonga. Les deux enregistrements malacologiques ont aussi en commun des valeurs de richesse relativement stables et un rapport J/A plus élevé durant le second réchauffement que durant le premier. Seules les valeurs des indices de diversité et d'équitabilité sont un peu plus variables à Nussloch, sûrement en raison d'une malacofaune plus riche qui inclut des taxons plus sensibles aux changements climato-environnementaux que ceux de Bourlon, qui sont présents dans quasiment toutes les malacofaunes glaciaires européennes (Ložek, 1964).

La structure uni-événementielle de l'enregistrement malacologique du gley de toundra de Curgies permet d'envisager trois corrélations possibles avec d'une part les gleys de toundra G3 ou G4, et d'autre part le pic d'abondance G2' de Nussloch (fig. 8). Ce dernier n'est toutefois pas associé à un gley de toundra clairement exprimé (Moine et al., 2008; Antoine et al., 2009). L'abondance maximale à Curgies n'est que deux fois moindre que celle de G2', et les deux pics sont surtout dus à des effectifs croissants des taxons hygrophiles et palustres. Or, une fois adaptée l'épaisseur du pic d'abondance de Curgies à celui de G2', seules les courbes du rapport J/A montrent une dynamique similaire. La mise en parallèle des deux maximas d'abondance avec ou sans adaptation de l'épaisseur du pic d'abondance de Curgies (non figuré; léger décalage vers le bas des courbes de Curgies) montre beaucoup plus de cohérence entre les variations des différents paramètres dans les deux sites. On constate que la faible épaisseur du pic d'abondance G2' à Nussloch, composé de seulement deux échantillons, rend difficile toute comparaison avec d'autres enregistrements malacologiques. Une éventuelle corrélation entre les enregistrements malacologiques du gley de toundra de Curgies et du pic d'abondance G2' n'est donc pas facile à mettre en évidence, mais elle n'est pas pour autant exclue.

La comparaison des paramètres malacologiques de Curgies avec les enregistrements malacologiques des gleys de toundra G3 et G4 est beaucoup plus probante (fig. 8). Même si les pics d'abondances à Nussloch sont environ cinq fois plus importants, ils ont une allure plus semblable à celui de Curgies, notamment celui de G4 plus asymétrique. En outre, la richesse et les indices de diversité montrent tous une forte décroissance, les rapports $\mathrm{J} / \mathrm{A}$ présentent un maxima synchrone, et les indices d'équitabilité montrent une baisse notable suivie d'une augmentation égale dans G3 et un peu plus importante dans G4. Les principales différences sont l'absence d'une augmentation de la proportion du taxon hygrophile Succinella oblonga durant G3, et la désynchronisation de ce signal entre Curgies et Nussloch dans G4. Cette désynchronisation est due à une croissance plus prédominante des effectifs des taxons hygrophiles et palustres à Curgies qu'à Nussloch au sommet de G4. Ceci peut s'expliquer par le contexte topographique plus plat à Curgies qui permet le développement de zones humides, alors qu'à Nussloch la position en sommet de dune de lœss implique des environnements mieux drainés moins favorables aux mollusques hygrophiles. La corrélation du gley de toundra de Curgies avec les gleys de toundra G3 ou G4 de Nussloch apparaît donc plus probable qu'avec le niveau associé au pic d'abondance G2'. Toutefois, même si la dynamique de l'ensemble des indices semble un peu plus favorable à G4, un contrôle chronologique indépendant reste néanmoins absolument nécessaire pour trancher entre ces trois possibilités.

Cette tentative inédite de corrélation d'enregistrements malacologiques entre les deux domaines «ouest» et «central» suggère que des malacofaunes contemporaines mais distantes peuvent être caractérisées par une dynamique similaire à l'échelle millénaire. Ceci a été montré également par les comparaisons des malacofaunes tardiglaciaires de séries d'Europe de l'ouest et centrale (Royaume-Uni, France, Pays-Bas, Allemagne) (Limondin-Lozouet, 2002). Seule la multiplication de tels enregistrements malacologiques associée à des datations radiométriques permettra de renforcer cette hypothèse. En cas de confirmation, l'approche spatiale à haute résolution temporelle de l'évolution des paléoenvironnements d'Europe de l'ouest au Pléniglaciaire supérieur, qui constitue à l'heure actuelle l'objectif de ces analyses malacologiques, serait donc envisageable. Cette tentative de corrélation inédite a également des implications stratigraphiques. En effet, malgré leur position stratigraphique similaire, les gleys de toundra de Curgies et de Bourlon ne seraient pas tous deux corrélables avec le gley de toundra de base de la stratigraphie synthétique des dépôts lœssiques du nord de la France si l'on tient compte de leurs malacofaunes. Des hiatus d'âge et de durée différents affecteraient donc les enregistrements sédimentaires du Pléniglaciaire supérieur du nord de la France, notamment dans sa moitié inférieure, ce qui expliquerait la relative simplicité de ces enregistrements sédimentaires par comparaison avec ceux de la vallée du Rhin (voir Contexte). La corrélation entre les gleys de toundra épais échantillonnés en colonnes continues à Curgies et à Bourlon avec la stratigraphie synthétique régionale n'est donc pas évidente. En outre, l'absence d'âge pour l'horizon cryoturbé du Santerre ne permet pas d'établir une corrélation avec les gleys de toundra de Curgies et de 

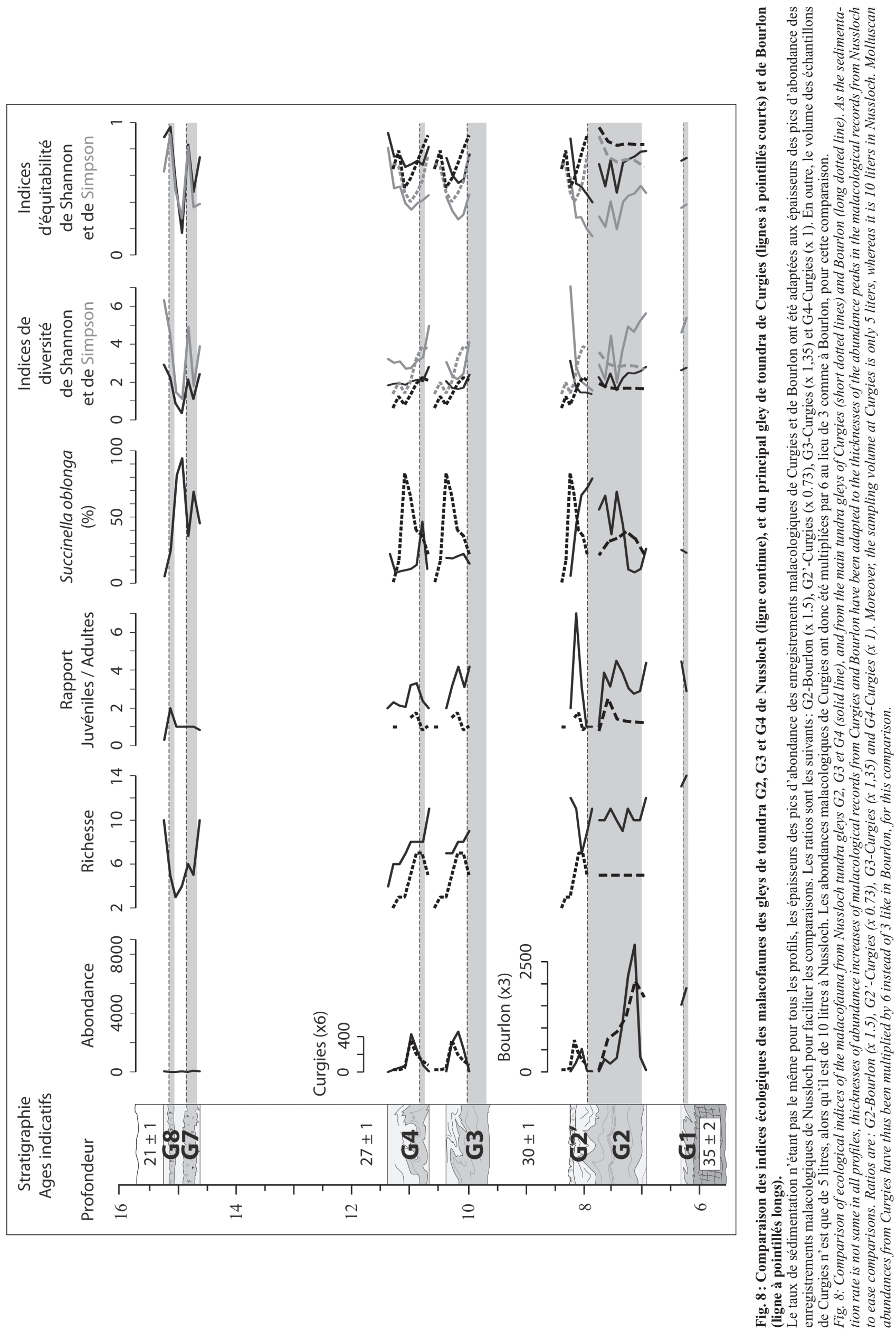
Bourlon malgré les corrélations proposées avec les gleys de toundra datés de Nussloch. Si les datations réalisées à Curgies étayent les corrélations établies sur la base de la malacofaune, alors le gley de toundra de Curgies serait plus récent que celui de Bourlon. Lâge numérique de l'horizon cryoturbé du Santerre n'étant pas connu, deux hypothèses de corrélation sont possibles (fig. 4): (1) le gley de toundra de Curgies serait corrélable avec 1'Horizon cryoturbé du Santerre (B), et celui de Bourlon au gley de toundra de base de la stratigraphie synthétique régionale $(\mathrm{A})$ ou à un autre gley de toundra intermédiaire, ou (2) le gley de toundra de Bourlon serait corrélé à l'horizon cryoturbé du Santerre (B) et celui de Curgies à un autre gley de toundra sus-jacent non-figuré. Les datations OSL en cours pour Curgies et celles associées aux analyses malacologiques à venir devraient permettre à l'avenir de lever ces questionnements.

\section{6 - CONCLUSION}

L'analyse de ces deux premiers enregistrements malacologiques à haute résolution dans le domaine malaco-biogéographique «ouest» a permis malgré le faible nombre d'échantillons composant chaque séquence :

- de renforcer le découpage et la caractérisation biogéographique du nord-ouest de l'Europe au Pléniglaciaire supérieur.

- de décrire pour la première fois la dynamique malacologique et paléoenvironnementale associée aux alternances lœss-gley dans ce domaine biogéographique.

- de mettre en évidence la variabilité des réponses environnementales associées aux alternances lœss-gley.

- de proposer, par analogie avec les résultats précédemment obtenus à Nussloch, une première tentative de corrélation entre les alternances lœss-gley décrites dans le nord de la France et les différentes phases des oscillations climatiques rapides stade-interstade (cycles de Dansgaard-Oeschger) qui jalonnent la dernière période glaciaire.

- de proposer des corrélations entre les gleys de toundra de Curgies et de Bourlon, et certains gley de toundra de Nussloch.

Les résultats obtenus posent toutefois de nouvelles questions concernant notamment la stratigraphie des dépôts de lœss du nord de la France (corrélation des profils, âge et durée des formations sédimentaires,...) auxquelles seule la multiplication de ce genre d'analyses malacologiques, et surtout la réalisation d'un cadre chronologique numérique, permettront d'apporter de nouveaux éléments de réponse.

\section{REMERCIEMENTS}

Le premier auteur tient à remercier ses co-auteurs ainsi que Sylvie Coutard, Emilie Goval et Jean-Luc Locht pour l'aide qu'ils lui ont apportée sur le terrain, notamment lors du prélèvement des échantillons malacologiques. Les auteurs remercient également Nicole LimondinLozouet et Jef Vandenberghe pour leurs commentaires constructifs ayant permis d'améliorer la version initiale de cet article.

\section{RÉFÉRENCES BIBLIOGRAPHIQUES}

ADAM W., 1960 - Mollusques. I. Mollusques terrestres et dulcicoles Institut royal des Sciences naturelles de Belgique, Bruxelles, $402 \mathrm{p}$.

ANDERSON R., 2005 - An annotated list of the non-marine Mollusca of Britain and Ireland. Journal of Conchology, 38 (6), 607-637.

ANTOINE P., 1988 - Contribution à l'étude des lœss du Pléistocène supérieur du bassin de la Somme. Revue archéologique de Picardie, 1-2, 25-44

ANTOINE P., 1990 - Chronostratigraphie et environnement du Paléolithique du bassin de la Somme. Publications du Centre d'études et de recherches préhistoriques, 2, 1-231.

ANTOINE P., 1991 - Nouvelles données sur la stratigraphie du Pléistocène supérieur de la France septentrionale, d'après les sondages effectués sur le tracé du TGV Nord. Publications du Centre d'études et de recherches préhistoriques, 3, 9-20.

ANTOINE P., LAUTRIDOU J.-P., SOMMÉ J., AUGUSTE P., AUFFRET J.-P., BAIZE S., CLET-PELLERIN M., COUTARD J.-P., DEWOLF Y., DUGUÉ O., JOLY F., LAIGNEL B. LAURENT M., LAVOLLE M., LEBRET P., LEFEBVRE D., LÉCOLLE F., LIMONDIN-LOZOUET N., MUNAUT A.-V., OZOUF J.-C., QUESNEL F., \& ROUSSEAU D.-D., 1998 - Les formations quaternaires de la France du Nord-Ouest: limites et corrélations. Quaternaire, 9 (3), 227-241.

ANTOINE P., ROUSSEAU D.-D., LAUTRIDOU J.-P., \& HATTÉ C., 1999 - Last interglacial-glacial climatic cycle in loess-paleoso successions of north-western France. Boreas, 28 (4), 551-563.

ANTOINE P., ROUSSEAU D.D., ZÖLLER L., LANG A., MUNAUT A.V., HATTÉ C., \& FONTUGNE M., 2001 - High-resolution record of the Last Interglacial-Glacial cycle in the loess palaeosol sequences of Nussloch Rhine Valley (Germany). Quaternary International, 76/77, 211-229.

ANTOINE P., 2002 - Les lœss en France et dans le nord-ouest européen. Revue Française de Géotechnique, 99, 3-21.

ANTOINE P., ROUSSEAU D.-D., HATTÉ C., ZÖLLER L., LANG A., FONTUGNE M., \& MOINE O., 2002 - Evénements éoliens rapides en contexte lœssique: l'exemple de la séquence du pléniglaciaire supérieur weichselien de Nussloch vallée du Rhin, Allemagne. Quaternaire, 13 (3), 199-208.

ANTOINE P., AUGUSTE P., BAHAIN J.-J., COUDRET P.,

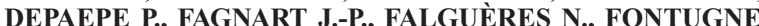
M., FRECHEN M., HATTÉ C., LAMOTTE A., LAURENT M., LIMONDIN-LOZOUET N., LOCHT J.-L., MERCIER N., MOIGNE A.-M., MUNAUT A.-V., \& PONEL P., 2003 - Paléoenvironnements pléistocènes et peuplements paléolithiques dans Ie bassin de la Somme nord de la France. Bulletin de la Société préhistorique française, 100 (1), 5-28.

ANTOINE P., CATT J., LAUTRIDOU J.-P., \& SOMMÉ J., 2003 The loess and coversands of northern France and Southern England. Journal of Quaternary Science, 18 (3/4), 309-318.

ANTOINE P., ROUSSEAU D.-D., MOINE O., KUNESCH S., HATTÉ C., LANGA., TISSOUX H., \& ZÖLLER L., 2009 - Rapid and cyclic aeolian deposition during the Last Glacial in European loess: a high-resolution record from Nussloch, Germany. Quaternary Science Reviews, 28 (25/26), 2955-2973.

BANK R. A., FALKNER G., \& VON PROSCHWITZ T., 2001 - CLECOM-PROJECT. A revised checklost of the non-marine Mollusca of Britain and Ireland. Heldia, 5, 41-72.

BIBUS E., 1980 - Zur Relief-, Boden- und Sedimententwicklung am unteren Mittelrhein. Frankfurter Geowissenschaftliche Arbeiten Serie D. Physische Geographie, 1, 1-295.

BIBUS E., FRECHEN M., KÖSEL M., \& RÄHLE W., 2007 - Das jungpleistozäne Lößprofil von Nußloch (SW-Wand) im Aufschluss der Heidelberger Zement AG. Eiszeitalter und Gegenwart, 56 (4), 227-255

BURACZYNSKI J., \& BUTRYM J., 1987 - Thermoluminescence stratigraphy of the loess in the southern Rhinegraben. Catena, Suppl. 9, 81-94.

DAVIES P., 1996 - The ecological status of Pupilla muscorum (Linné) in Holocene overbank alluvium at Kingsmead Bridge, Wiltshire. Journal of Conchology, 35 (6), 467-471. 
FAGNART J.-P., COUDRET P., ANTOINE P., avec la collaboration de VALLIN L., SELLIER N. \& MASSON B., (sous presse) - Le Paléolithique supérieur ancien dans le Nord de la France. Bulletin de la Société Préhistorique de France.

FALKNER G., RIPKEN T.E.J., \& FALKNER M., 2002 - Mollusques continentaux de France. Liste de référence annotée et bibliographie. Patrimoines naturels, 52, 1-350.

FRECHEN M., 1999 - Upper Pleistocene loess stratigraphy in Southern Germany. Quaternary Science Reviews, 18 (2), 243-269.

FRECHEN M., ZANDER A., CÍLEK V., \& LOŽEK V., 1999 - Loess chronology of the Last Interglacial/Glacial cycle in Bohemia and Moravia, Czech Republic. Quaternary Science Reviews, 18 (13), 1467-1493.

FRECHEN M., VAN VLIET-LANOË B., \& VAN DEN HAUTE P., 2001 - The Upper Pleistocene loess record at Harmignies/Belgium - high resolution terrestrial archive of climate forcing. Palaeogeography, Palaeoclimatology, Palaeoecology, 173 (3/4), 175-195.

FRECHEN M., OCHES E. A., \& KOHFELD K.E., 2003 - Loess in Europe-mass accumulation rates during the Last Glacial period. Quaternary Science Reviews, 22 (18/19), 1835-1857.

FRENCH H.M., 2007 - The periglacial environment. Wiley, Chichester, $458 \mathrm{p}$.

GERMAIN L., 1930 - Mollusques terrestres et fluviatiles. Librairie de la Faculté des Sciences, Paris, 477 p.

GULLENTOPS F., 1981 - About the climate of the last glaciation in NW-Europe. In Ouaternary climatic variations in a Milankovitch Perspective, 2th of June 1981. Université Catholique de Louvain, Louvain, 1-5.

HAASE D., FINK J., HAASE G., RUSKE R., PÉCSI M., RICHTER H., ALTERMANN M., \& JÄGER K.-D., 2007 - Loess in Europeits spatial distribution based on a European Loess Map, scale 1:2,500,000. Quaternary Science Reviews, 26 (9/10), 1301-1312.

HAESAERTS P., \& VAN VLIET-LANOË B., 1974 - Compte rendu de l'excursion du 25 mai 1974 consacrée à la stratigraphie des limons aux environs de Mons. Annales de la Société Géologique de Belgique, 97, 547-560.

HAESAERTS P., 1980 - Stratigraphie des dépôts limoneux du Pléistocène supérieur de Moyenne Belgique: essai de zonation paléoclimatique. Bulletin de l'Association Française pour l'Etude du Quaternaire, N.S. 1, 165-173.

HAESAERTS P., JUVIGNÉ E., KUYL O., MÜCHER H., \& ROEBROEKS W., 1981 - Compte rendu de l'excursion du 13 juin 1981, en Hesbaye et au Limbourg Néerlandais, consacrée à la chronostratigraphie des lœss du Pléistocène supérieur. Annales de la Société Géologique de Belgique, 104, 223-240.

HAESAERTS P., \& VAN VLIET-LANOË B., 1981 - Phénomènes périglaciaires et sols fossiles observés à Maisières-Canal, à Harmignies et à Rocourt. Biuletyn Peryglacjalny, 28, 208-216.

HAESAERTS P., 1985 - Les loess du Pléistocène supérieur en Belgique; comparaisons avec les séquences d'Europe centrale. Bulletin de l'Association Française pour l'Etude du Quaternaire, 22 (2/3), 105-115.

HAESAERTS P., BORZIAK I., CHIRICA V., DAMBLON F., KOULAKOVSKA L., \& VAN DER PLICHT J., 2003 - The East Carpathian loess record: a reference for the Middle and Late Pleniglacial stratigraphy in Central Europe. Quaternaire, 14 (3), 163-188.

HATTÉ C., \& GUIOT J., 2005 - Palaeoprecipitation reconstruction by inverse modelling using the isotopic signal of loess organic matter: application to the Nußloch loess sequence Rhine Valley, Germany. Climate Dynamics, 25 (2), 315-327.

HUIJZER A.S., \& 1993 - Cryogenic microfabrics and macrostructures: interrelations, processes and paleoclimatic significance. $\mathrm{Ph}$. D. thesis, Vrije Universiteit, Amsterdam, 245 p.

KERNEY M.P., 1971 - A Middle Weichselian deposit at Halling, Kent. Proceedings of the Geologists' Association, 82, 1-10.

KERNEY M.P., CAMERON R.A.D., \& JUNGBLUTH J.H., 1983 Die Landschnecken Nord- und Mitteleuropas. Paul Parey, Hamburg, $384 \mathrm{p}$.

KUKLA G., 1977 - Pleistocene land-sea correlations. I. Europe. Earth Science Reviews, 13 (4), 307-374.

LANG A., HATTÉ C., ROUSSEAU D.-D., ANTOINE P., FONTUGNE M., ZÖLLER L., \& HAMBACH U., 2003 - High resolutions chronologies for loess: comparing AMS-14C and optical dating results. Quaternary Science Reviews, 22 (10/13), 953- 959.

LIKHACHEV I.M., \& RAMMEL'MEIER E.S., 1962 - Terrestrial Mollusks of the Fauna of the U.S.S.R. Oldbourne Press, Jerusalem, $574 \mathrm{p}$.

LIMONDIN-LOZOUET N., \& ANTOINE P., 2001 - Palaeoenvironmental changes inferred from malacofaunas in the Lateglacial and early Holocene fluvial sequence at Conty, northern France. Boreas, 30 (2), 148-164.
LIMONDIN-LOZOUET N., 2002 - Impact des oscillations climatiques du Tardiglaciaire sur l'évolution des malacofaunes de fond de vallée en Europe du Nord-Ouest. Annales littéraires de l'Université de Besançon, 730, 45-51.

LOCHT J.-L., ANTOINE P., BAHAIN J.-J., DWRILA G., RAYMOND P., LIMONDIN-LOZOUET N., GAUTHIER A., DEBENHAM N., FRECHEN M., ROUSSEAU D.-D., HATTÉ C., HAESAERTS P., \& METSDAGH H., 2003 - Le gisement paléolithique moyen et les séquences pléistocènes de Villiers-Adam (Val d'Oise). Chronostratigraphie, environnements et implantations humaines. Gallia Préhistoire, 45, 1-111.

LOCHT J.-L., ANTOINE P., AUGUSTE P., BAHAIN J.-J., DEBENHAM N., FALGUËRES C., FARKH S., \& TISSOUX H., 2006 - La séquence lœssique pléistocène supérieur de Savy Aisne (France): stratigraphie, datations et occupations paléolithiques. Quaternaire, 17 (3), 269-275.

LOŽEK V., 1964 - Quartärmollusken der Tschechoslowakei. Rozpravy Ustredniho ustuvu geologického, 31, 1-374.

MAZENOT G., 1951 - Découverte, à Lyon, d'un faciès lacustre du loess récent. Revue de Géographie de Lyon, 26 (2), 190-202.

MEIJS E.P.M., 2002 - Loess stratigraphy in Dutch and Belgian Limburg. Eiszeitalter und Gegenwart, 51 (1), 114-130.

MOINE O., ROUSSEAU D.-D., \& ANTOINE P., 2005 - Terrestrial molluscan records of Weichselian Lower to Middle Pleniglacial climatic changes from the Nussloch loess series Rhine Valley (Germany): the impact of local factors. Boreas, 34 (3), 363-380.

MOINE O., 2008 - West-European malacofauna from loess deposits of the Weichselian Upper Pleniglacial: compilation and preliminary analysis of the database. Quaternaire, 19 (1), 11-29.

MOINE O., ROUSSEAU D.-D., \& ANTOINE P., 2008 - The impact of Dansgaard-Oeschger cycles on the loessic environment and malacofauna of Nussloch (Germany) during the Upper Weichselian. Quaternary Research, 70 (1), 91-104.

PUISSÉGUR J.-J., 1976 - Mollusques continentaux quaternaires de Bourgogne. Significations stratigraphiques et climatiques. Rapports avec d'autres faunes boréales de France. Mémoires Géologiques de l'Université de Dijon, 3, 1-241.

PYE K., 1995 - The nature, origin and accumulation of loess. Quaternary Science Reviews, 14 (7/8), 653-667.

REIMER P.J., BAILLIE M.G.L., BARD E., BAYLISS A., BECK J.W., BLACKWELL P.G., BRONK RAMSEY C., BUCK C.E., BURR G.S., EDWARDS R.L., FRIEDRICH M., GROOTES P.M., GUILDERSO T.P., HAJDAS I., HEATON T.J., HOGG A.G., HUGHEN K.A., KAISER K.F., KROMER B., MCCORMAC F.G., MANNING S.W., REIMER R.W., RICHARDS D.A., SOUTHON J.R., TALAMO S., TURNEY C.S.M., VAN DER PLICHT J., \& WEYHENMEYER C.E., 2009 - IntCal09 and Marine 09 radiocarbon age calibration curves, 0-50,000 years cal BP. Radiocarbon, 51 (4), 1111-1150.

REMY H., 1969 - Würmzeitliche Molluskenfaunen aus Lößserien des Rheingaues und der nördlichen Rheinhessens. Notizblatt des Hessischen Landesamtes für Bodenforschung zu Wiesbaden, 97, 98-116.

ROCROI J.-P., BICHAIN J.-M., \& GARGOMINY O., 2007 - De Trichia à Trochulus Gastropoda, Hygromiidae. MalaCo, 4, 150.

ROUSSEAU D.-D., \& LAURIN B., 1984 - Variations de Pupilla muscorum L. (Gastropoda) dans le Quaternaire d'Achenheim (Alsace) : une analyse de l'interaction entre espèce et milieu. Géobios, Mémoire spécial 8, 349-355.

ROUSSEAU, D.-D., 1997 - The weight of internal and external constraints on Pupilla muscorum L. (Gastropoda: Stylommatophora) during the Quaternary in Europe. In B. Huntley, W. Cramer, A.V. Morgan, H.C. Prentice, \& J.R.M. Allen, Past and Future Environmental Changes: The Spatial and Evolutionary Responses of Terrestrial Biota. NATO ASI Series, I47. Springer Verlag, Heidelberg, 303-318.

ROUSSEAU, D.-D., ZÖLLER, L., \& VALET, J.-P., 1998 - Late Pleistocene climatic variations at Achenheim, France, based on a magnetic susceptibility and TL chronology of loess. Quaternary Research, 49 (3), 255-263.

ROUSSEAU D.-D., ANTOINE P., HATTÉ C., LANG A., ZÖLLER L., FONTUGNE M., BEN OTHMAN D., LUCK J.-M., MOINE O., LABONNE M., BENTALEB I., \& JOLLY D., 2002 - Abrupt millennial climatic changes from Nussloch (Germany) Upper Weichselian eolian records during the Last Glaciation. Quaternary Science Reviews, 21 (14/15), 1577-1582.

ROUSSEAU D.-D., SIMA A., ANTOINE P., HATTÉ C., LANG A., \& ZÖLLER L., 2007 - Link between European and North Atlantic abrupt climate changes over the last glaciations. Geophysical Research Letters, 34, L22713, doi:10.1029/2007GL031716.

SCHIERMEYER J., 2002 - Würmzeitliche Lößmollusken aus der Eifel. Thèse de Doctorat, Universität Düsseldorf, Düsseldorf, 125 p. 
SCHIRMER W., 2000 - Eine Klimakurve des Oberpleistozäns aus dem rheinischen Löss. Eiszeitalter und Gegenwart, 50 (1), 25-49.

SEMMEL A., 1968 - Studien über den Verlauf jungpleistozäner Formung in Hessen. Frankfurter geographische Hefte, 45, 1-133.

SHANNON C.E., \& WEAVER W., 1949 - The mathematical theory of communication. The University of Illinois Press, Urbana, $125 \mathrm{p}$

SIMPSON E.H., 1949 - Measurement of diversity. Nature 163 (4148), 688-689.

TISSOUX H., VALLADAS H., VOINCHET P., REYSS J.-L., MERCIER N., FALGUÊRES C., BAHAIN J.-J., ZÖLLER L., \& ANTOINE, P., 2010 - OSL and ESR studies of aeolian quartz from the Upper Pleistocene loess sequence of Nussloch (Germany). Quaternary Geochronology, 5 (2/3), 131-136.

VAN DEN HAUTE P., VANCRAEYNEST L., \& DE CORTE, F, 1998 - The Late Pleistocene loess deposits and paleosols of eastern Belgium: new TL age determinations. Journal of Quaternary Science, 13 (5), 487-497.

VAN VLIET-LANOË B., 1985 - Frost effects in soils. In J. Boardman (ed.), Soils and Quaternary landscape evolution. John Wiley \& Sons, Chichester, 117-158.

VAN VLIET-LANOË B., 1992 - Le niveau à langues de Kesselt, horizon repère de la stratigraphie du Weichsélien supérieur européen : signification paléoenvironnementale et paléoclimatique. Bulletin de la Société Géologique de France, 160, 35-44.
VANDENBERGHE J., HUIJZER B.S., MÜCHER H., \& LAAN W., 1998 - Short climatic oscillations in a western European loess sequence (Kesselt, Belgium). Journal of Quaternary Science, 13 (5), 471-485.

VANDENBERGHE J., \& NUGTEREN G., 2001 - Rapid climatic changes recorded in loess successions. Global and Planetary Change, 28 (1/4), 1-9

VANDENBERGHE J., LOWE J., COOPE R., LITT T., \& ZÖLLER L., 2004 - Climatic and environmental variability in the mid-latitude Europe sector during the last interglacial-glacial cycle. In R.W Battarbee (ed.), Past climate variability through Europe and Africa. Springer, Dordrecht, 393-416.

ZILCH A., \& JAECKEL S.G.A., 1962 - Mollusken. Quelle Meyer Leipzig, $293 \mathrm{p}$

ZÖLLER L., ROUSSEAU, D.-D., JÄGER K.-D., \& KUKLA G 2004 - Last interglacial, Lower and Middle Weichselian - a comparative study from the Upper Rhine and Thuringian loess areas. Zeitschrift für Geomorphologie, 48 (1), 1-24. 